\title{
Fucosylated chondroitin sulfate 9-18 oligomers exhibit molecular size-independent antithrombotic activity during circulating in the \\ blood
}

Lufeng Yan $\star^{\text {, }}$, Danli Wang $\star$, Yanlei Yu ${ }^{\S}$, Fuming Zhang $\S$, Xingqian Ye ${ }^{\star}$, Robert J. Linhardt ${ }^{\S 1}$, Shiguo Chen $\star^{2}$

$\$$ College of Biosystems Engineering and Food Science, National-Local Joint Engineering Laboratory of Intelligent Food Technology and Equipment, Zhejiang Key Laboratory for Agro-Food Processing, Integrated Research Base of Southern Fruit and Vegetable Preservation Technology, Zhejiang International Scientific and Technological Cooperation Base of Health Food Manufacturing and Quality Control, Zhejiang University, Hangzhou 310058

$\S$ Center for Biotechnology \& Interdisciplinary Studies and Department of Chemistry \& Chemical Biology, Rensselaer Polytechnic Institute, Biotechnology Center 4005, Troy, NY 12180, United States

1 Corresponding author. Center for Biotechnology \& Interdisciplinary Studies and Department of Chemistry \& Chemical Biology, Rensselaer Polytechnic Institute, Biotechnology Center 4005, Troy, NY 12180, United States

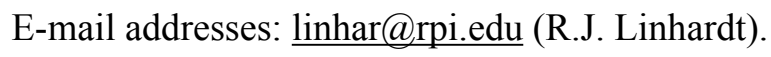

Telephone number: $+1-518-276-3404$

Fax number: +1-518-276 -3405

${ }^{2}$ Corresponding author. College of Biosystems Engineering and Food Science, Zhejiang University, Hangzhou 310058, China

E-mail addresses: chenshiguo210@163.com (S. Chen).

Telephone number: +86-13116780577

Fax number: +86-571-88982151 
Table S1 ${ }^{1} \mathrm{H}$ and ${ }^{13} \mathrm{C}$ chemical shifts of FCS-Ib oligomers.
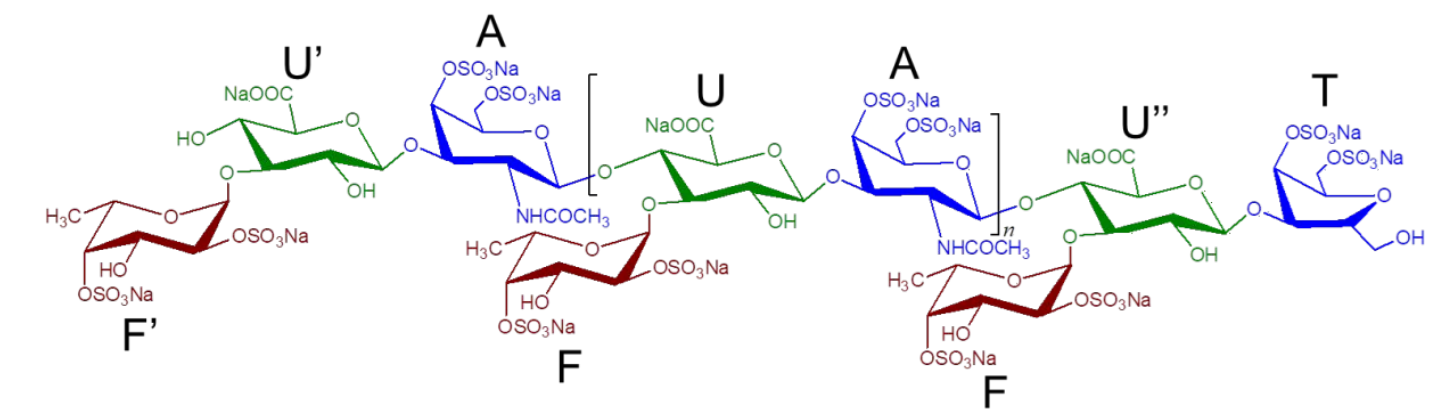

\begin{tabular}{|c|c|c|c|c|c|c|c|c|}
\hline & \multicolumn{8}{|c|}{ Chemical shift (ppm) } \\
\hline & $\begin{array}{l}\mathrm{H}-1 \\
(\mathrm{C}-1)\end{array}$ & $\begin{array}{l}\mathrm{H}-2 \\
(\mathrm{C}-2)\end{array}$ & $\begin{array}{l}\mathrm{H}-3 \\
(\mathrm{C}-3)\end{array}$ & $\begin{array}{l}\mathrm{H}-4 \\
(\mathrm{C}-4)\end{array}$ & $\begin{array}{l}\mathrm{H}-5 \\
(\mathrm{C}-5)\end{array}$ & $\begin{array}{l}\text { H-6 } \\
(\mathrm{C}-6)\end{array}$ & $\mathrm{CH}_{3}$ & $\mathrm{C}=\mathrm{O}$ \\
\hline \multicolumn{9}{|c|}{ FCS-Ib 6-mer $(n=0)$} \\
\hline $\mathbf{F}$ & 5.68 & 4.47 & 4.13 & 4.85 & 4.89 & 1.36 & - & - \\
\hline Fuc $2,4 \mathrm{~S}(\alpha)$ & $(97.2)$ & $(75.6)$ & $(67.1)$ & $(81.7)$ & $(67.0)$ & $(16.4)$ & & \\
\hline $\mathrm{F}^{\prime}$ & 5.56 & 4.43 & 4.15 & 4.67 & 4.53 & 1.24 & - & - \\
\hline Fuc2,4S $(\alpha)$ & $(97.3)$ & $(75.7)$ & $(67.0)$ & $(81.4)$ & $(66.7)$ & $(16.2)$ & & \\
\hline U' & 4.52 & 3.58 & 3.64 & 3.70 & 3.85 & - & - & - \\
\hline $\operatorname{Glc} A(\beta)$ & $(104.2)$ & $(73.4)$ & $(82.0)$ & $(70.3)$ & $(76.5)$ & & & $(175.6)$ \\
\hline U’" & 4.53 & 3.70 & 3.79 & 3.98 & 3.83 & - & - & - \\
\hline $\operatorname{GlcA}(\beta)$ & $(102.4)$ & $(74.1)$ & $(77.6)$ & $(75.9)$ & $(76.4)$ & & & $(174.2)$ \\
\hline $\mathbf{A}$ & 4.57 & 4.06 & 3.98 & 4.83 & 3.97 & $4.27 / 4.16$ & 2.04 & - \\
\hline GalNAc4,6S( $\beta)$ & $(100.6)$ & $(51.9)$ & $(77.0)$ & $(76.8)$ & $(72.4)$ & $(67.8)$ & $(23.1)$ & $(174.4)$ \\
\hline $\mathbf{T}$ & $3.69 / 3.81$ & 4.13 & 4.52 & 5.04 & 4.51 & $4.35 / 4.18$ & - & - \\
\hline anTal-ol4,6S & $(61.4)$ & $(81.4)$ & $(77.0)$ & $(78.0)$ & $(78.0)$ & $(68.6)$ & & \\
\hline \multicolumn{9}{|c|}{ FCS-Ib 9-mer $(n=1)$} \\
\hline F & 5.68 & 4.47 & 4.12 & 4.86 & 4.87 & 1.35 & - & - \\
\hline Fuc $2,4 \mathrm{~S}(\alpha)$ & $(97.0)$ & $(75.6)$ & $(67.1)$ & $(81.7)$ & $(67.0)$ & $(16.4)$ & & \\
\hline F' & 5.56 & 4.44 & 4.15 & 4.67 & 4.53 & 1.23 & - & - \\
\hline Fuc $2,4 \mathrm{~S}(\alpha)$ & $(97.3)$ & $(75.7)$ & $(67.1)$ & $(81.4)$ & $(66.8)$ & $(16.2)$ & & \\
\hline $\mathbf{U}^{\prime}$ & 4.52 & 3.58 & 3.64 & 3.71 & 3.86 & - & - & - \\
\hline $\operatorname{GlcA}(\beta)$ & (104.3) & $(73.4)$ & $(82.0)$ & $(70.3)$ & $(76.4)$ & & & $(175.6)$ \\
\hline $\mathbf{U}$ & 4.49 & 3.63 & 3.80 & 3.99 & 3.85 & - & - & - \\
\hline $\operatorname{GlcA}(\beta)$ & $(104.5)$ & $(74.1)$ & $(77.6)$ & $(75.8)$ & $(76.2)$ & & & $(174.0)$ \\
\hline $\mathbf{U}$ & 4.53 & 3.70 & 3.77 & 3.96 & 3.82 & - & - & - \\
\hline $\operatorname{Glc} A(\beta)$ & $(102.3)$ & $(74.1)$ & $(77.6)$ & $(75.8)$ & $(76.3)$ & & & (173.8) \\
\hline A & 4.57 & 4.04 & 3.96 & 4.81 & 3.97 & $4.25 / 4.16$ & 2.03 & - \\
\hline GalNAc4,6S $(\beta)$ & $(100.6)$ & $(51.9)$ & $(77.0)$ & $(76.8)$ & $(72.4)$ & $(67.9)$ & $(23.0)$ & (174.3) \\
\hline $\mathbf{T}$ & $3.69 / 3.80$ & 4.13 & 4.52 & 5.03 & 4.51 & $4.35 / 4.17$ & - & - \\
\hline anTal-ol4,6S & $(61.5)$ & $(81.5)$ & $(77.1)$ & $(77.9)$ & $(78.0)$ & $(68.6)$ & & \\
\hline \multicolumn{9}{|c|}{ FCS-Ib 12-mer $(n=2)$} \\
\hline $\mathbf{F}$ & 5.69 & 4.47 & 4.12 & 4.86 & 4.86 & 1.35 & - & - \\
\hline Fuc $2,4 \mathrm{~S}(\alpha)$ & $(96.9)$ & $(75.7)$ & $(67.2)$ & $(81.6)$ & $(67.0)$ & $(16.4)$ & & \\
\hline $\mathrm{F}^{\prime}$ & 5.56 & 4.43 & 4.15 & 4.67 & 4.53 & 1.24 & - & - \\
\hline Fuc $2,4 \mathrm{~S}(\alpha)$ & $(97.3)$ & $(75.8)$ & $(67.2)$ & $(81.5)$ & $(66.8)$ & $(16.3)$ & & \\
\hline U' & 4.52 & 3.58 & 3.64 & 3.71 & 3.87 & - & - & - \\
\hline $\operatorname{GlcA}(\beta)$ & (104.4) & $(73.5)$ & $(82.1)$ & $(70.4)$ & $(76.4)$ & & & $(175.6)$ \\
\hline $\mathbf{U}$ & 4.49 & 3.62 & 3.79 & 3.99 & 3.85 & - & - & - \\
\hline $\operatorname{Glc} A(\beta)$ & $(104.5)$ & $(74.2)$ & $(77.6)$ & $(75.7)$ & $(76.2)$ & & & $(174.0)$ \\
\hline U', & 4.53 & 3.70 & 3.78 & 3.97 & 3.81 & - & - & - \\
\hline $\operatorname{Glc} A(\beta)$ & $(102.6)$ & $(74.1)$ & $(77.6)$ & $(75.9)$ & $(76.2)$ & & & $(173.8)$ \\
\hline A & 4.57 & 4.04 & 3.96 & 4.81 & 3.97 & $4.25 / 4.16$ & 2.03 & - \\
\hline GalNAc4,6S( $\beta)$ & $(100.6)$ & $(51.9)$ & $(77.1)$ & $(76.8)$ & $(72.4)$ & $(67.8)$ & $(23.1)$ & $(174.2)$ \\
\hline $\mathbf{T}$ & $3.68 / 3.80$ & 4.14 & 4.52 & 5.04 & 4.51 & $4.35 / 4.18$ & - & - \\
\hline anTal-ol4,6S & $(61.5)$ & $(81.5)$ & $(77.2)$ & $(78.0)$ & $(77.9)$ & $(68.6)$ & & \\
\hline \multicolumn{9}{|c|}{ FCS-Ib 15-mer $(n=3)$} \\
\hline $\mathbf{F}$ & 5.69 & 4.47 & 4.12 & 4.85 & 4.86 & 1.34 & - & - \\
\hline Fuc $2,4 \mathrm{~S}(\alpha)$ & $(96.9)$ & $(75.6)$ & $(67.2)$ & $(81.7)$ & $(66.9)$ & $(16.4)$ & & \\
\hline $\mathbf{F}^{\prime}$ & 5.56 & 4.43 & 4.14 & 4.67 & 4.53 & 1.23 & - & - \\
\hline
\end{tabular}




\begin{tabular}{|c|c|c|c|c|c|c|c|c|}
\hline Fuc $2,4 \mathrm{~S}(\alpha)$ & $(97.3)$ & (75.8) & $(67.1)$ & (81.4) & $(66.8)$ & $(16.2)$ & & \\
\hline U' & 4.52 & 3.58 & 3.63 & 3.71 & 3.86 & - & - & - \\
\hline $\operatorname{GlcA}(\beta)$ & $(104.3)$ & $(73.5)$ & $(82.0)$ & $(70.2)$ & $(76.3)$ & & & (175.6) \\
\hline $\mathbf{U}$ & 4.49 & 3.62 & 3.79 & 3.99 & 3.83 & - & - & - \\
\hline $\operatorname{GlcA}(\beta)$ & $(104.5)$ & $(74.2)$ & $(77.6)$ & $(75.8)$ & $(76.2)$ & & & $(174.0)$ \\
\hline $\mathbf{U}^{\prime}$ & 4.53 & 3.70 & 3.77 & 3.96 & 3.81 & - & - & - \\
\hline $\operatorname{Glc} A(\beta)$ & $(102.2)$ & (74.1) & (77.6) & $(75.8)$ & $(76.1)$ & & & (173.8) \\
\hline $\mathbf{A}$ & 4.57 & 4.03 & 3.95 & 4.81 & 3.96 & $4.26 / 4.16$ & 2.03 & - \\
\hline GalNAc4,6S( $\beta)$ & (100.6) & (51.9) & $(77.1)$ & $(76.7)$ & $(72.3)$ & $(67.8)$ & $(23.1)$ & (174.3) \\
\hline $\begin{array}{l}\mathbf{T} \\
\text { anTal-ol4,6S }\end{array}$ & $\begin{array}{l}3.68 / 3.80 \\
(61.2)\end{array}$ & $\begin{array}{l}4.13 \\
(81.5)\end{array}$ & $\begin{array}{l}4.52 \\
(77.0)\end{array}$ & $\begin{array}{l}5.04 \\
(77.9)\end{array}$ & $\begin{array}{l}4.51 \\
(78.0)\end{array}$ & $\begin{array}{l}4.35 / 4.17 \\
(68.6)\end{array}$ & - & - \\
\hline \multicolumn{9}{|c|}{ FCS-Ib 18-mer $(n=4)$} \\
\hline $\mathbf{F}$ & 5.69 & 4.47 & 4.11 & 4.86 & 4.86 & 1.34 & - & - \\
\hline Fuc $2,4 \mathrm{~S}(\alpha)$ & $(96.9)$ & $(75.6)$ & $(67.2)$ & (81.7) & (66.9) & (16.4) & & \\
\hline F' & 5.56 & 4.43 & 4.15 & 4.66 & 4.53 & 1.24 & - & - \\
\hline Fuc $2,4 \mathrm{~S}(\alpha)$ & $(97.3)$ & (75.7) & $(67.2)$ & (81.4) & (66.8) & $(16.2)$ & & \\
\hline U' & 4.52 & 3.58 & 3.63 & 3.71 & 3.84 & - & - & - \\
\hline $\operatorname{GlcA}(\beta)$ & $(104.3)$ & (73.4) & $(82.0)$ & $(70.3)$ & (76.3) & & & (175.6) \\
\hline $\mathbf{U}$ & 4.48 & 3.62 & 3.79 & 3.97 & 3.80 & - & - & - \\
\hline $\operatorname{Glc} A(\beta)$ & $(104.5)$ & (74.2) & (77.6) & $(75.8)$ & $(76.2)$ & & & $(174.0)$ \\
\hline U', & 4.53 & 3.69 & 3.79 & 3.95 & 3.80 & - & - & - \\
\hline $\operatorname{GlcA}(\beta)$ & $(102.4)$ & (74.1) & (77.6) & (75.8) & (76.2) & & & (173.8) \\
\hline $\mathbf{A}$ & 4.57 & 4.03 & 3.94 & 4.79 & 3.96 & $4.25 / 4.15$ & 2.03 & - \\
\hline GalNAc4,6S $(\beta)$ & $(100.6)$ & $(51.9)$ & $(77.2)$ & (76.8) & (72.3) & $(67.8)$ & $(23.1)$ & (174.3) \\
\hline $\mathbf{T}$ & $3.68 / 3.80$ & 4.13 & 4.52 & 5.04 & 4.51 & $4.35 / 4.17$ & - & - \\
\hline anTal-ol4,6S & $(61.5)$ & $(81.4)$ & (77.0) & (77.9) & $(78.0)$ & $(68.6)$ & & \\
\hline
\end{tabular}


Table S2 ${ }^{1} \mathrm{H}$ and ${ }^{13} \mathrm{C}$ chemical shifts of FCS- $P g$ oligomers.

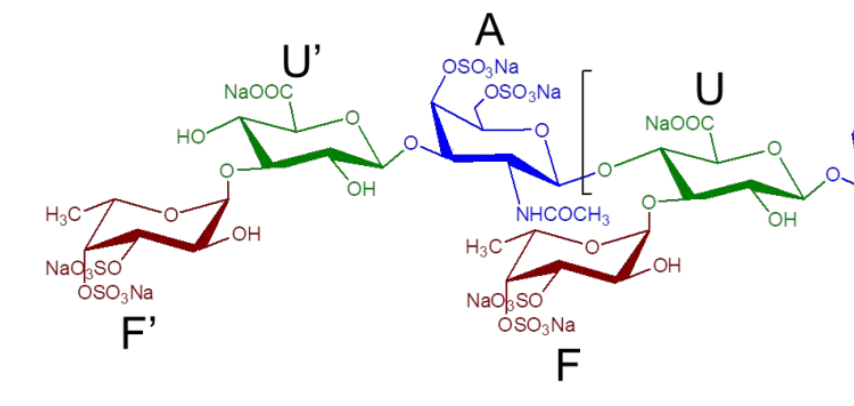

\begin{tabular}{|c|c|c|c|c|c|c|c|c|}
\hline & \multicolumn{8}{|c|}{ Chemical shift (ppm) } \\
\hline & $\begin{array}{l}\mathrm{H}-1 \\
(\mathrm{C}-1)\end{array}$ & $\begin{array}{l}\mathrm{H}-2 \\
(\mathrm{C}-2)\end{array}$ & $\begin{array}{l}\mathrm{H}-3 \\
(\mathrm{C}-3)\end{array}$ & $\begin{array}{l}\mathrm{H}-4 \\
(\mathrm{C}-4)\end{array}$ & $\begin{array}{l}\mathrm{H}-5 \\
(\mathrm{C}-5) \\
\end{array}$ & $\begin{array}{l}\text { H-6 } \\
(\mathrm{C}-6)\end{array}$ & $\mathrm{CH}_{3}$ & $\mathrm{C}=\mathrm{O}$ \\
\hline \multicolumn{9}{|c|}{ FCS-Pg 6-mer $(n=0)$} \\
\hline $\mathbf{F}$ & 5.32 & 3.92 & 4.48 & 4.93 & 4.71 & 1.36 & - & - \\
\hline Fuc $3,4 \mathrm{~S}(\alpha)$ & $(99.9)$ & $(66.9)$ & $(75.9)$ & $(79.9)$ & $(67.2)$ & $(16.5)$ & & \\
\hline $\mathrm{F}^{\prime}$ & 5.34 & 3.93 & 4.58 & 4.86 & 4.48 & 1.22 & - & - \\
\hline Fuc $3,4 \mathrm{~S}(\alpha)$ & $(99.4)$ & $(66.9)$ & $(75.7)$ & $(79.7)$ & $(66.9)$ & $(16.2)$ & & \\
\hline $\mathbf{U}^{\prime}$ & 4.54 & 3.58 & 3.64 & 3.73 & 3.90 & - & - & - \\
\hline $\operatorname{GlcA}(\beta)$ & $(104.0)$ & $(73.3)$ & $(81.3)$ & $(70.0)$ & $(75.6)$ & & & $(175.4)$ \\
\hline U', & 4.59 & 3.70 & 3.79 & 4.10 & 3.98 & - & - & - \\
\hline $\operatorname{Glc} A(\beta)$ & $(102.0)$ & $(73.4)$ & $(79.9)$ & $(75.7)$ & $(76.1)$ & & & $(173.3)$ \\
\hline A & 4.57 & 4.06 & 3.98 & 4.82 & 4.02 & $4.28 / 4.23$ & 2.03 & - \\
\hline GalNAc4,6S( $\beta)$ & $(100.4)$ & $(52.0)$ & $(76.6)$ & $(76.5)$ & $(72.4)$ & $(67.7)$ & $(22.9)$ & $(173.3)$ \\
\hline $\begin{array}{l}\mathbf{T} \\
\text { anTal-ol4,6S }\end{array}$ & $\begin{array}{l}3.67 / 3.79 \\
(61.4)\end{array}$ & $\begin{array}{l}4.13 \\
(81.3)\end{array}$ & $\begin{array}{l}4.51 \\
(76.7)\end{array}$ & $\begin{array}{l}5.03 \\
(77.8)\end{array}$ & $\begin{array}{l}4.49 \\
(77.9)\end{array}$ & $\begin{array}{l}4.33 / 4.17 \\
(68.4)\end{array}$ & - & - \\
\hline \multicolumn{9}{|c|}{ FCS-Pg 9-mer $(n=1)$} \\
\hline $\mathbf{F}$ & 5.32 & 3.93 & 4.49 & 4.94 & 4.74 & 1.37 & - & - \\
\hline Fuc3,4S $(\alpha)$ & (99.9) & $(66.9)$ & $(75.9)$ & $(79.9)$ & $(67.2)$ & $(16.5)$ & & \\
\hline F' & 5.35 & 3.95 & 4.59 & 4.87 & 4.49 & 1.23 & - & - \\
\hline Fuc3,4S $(\alpha)$ & $(99.5)$ & $(66.9)$ & $(75.8)$ & $(79.7)$ & $(66.9)$ & $(16.2)$ & & \\
\hline U' & 4.56 & 3.59 & 3.63 & 3.74 & 3.90 & - & - & - \\
\hline $\operatorname{GlcA}(\beta)$ & $(104.0)$ & $(73.4)$ & $(81.3)$ & $(70.1)$ & $(75.5)$ & & & $(175.5)$ \\
\hline $\mathbf{U}$ & 4.54 & 3.63 & 3.78 & 4.12 & 3.94 & - & - & - \\
\hline $\operatorname{Glc} A(\beta)$ & $(104.0)$ & $(73.7)$ & $(79.8)$ & $(75.5)$ & $(75.9)$ & & & $(173.2)$ \\
\hline U', & 4.60 & 3.71 & 3.75 & 4.08 & 4.01 & - & - & - \\
\hline $\operatorname{GlcA}(\beta)$ & $(102.0)$ & $(73.5)$ & $(79.6)$ & $(75.5)$ & $(75.7)$ & & & $(173.0)$ \\
\hline $\mathbf{A}$ & 4.56 & 4.06 & 3.98 & 4.81 & 4.03 & $4.30 / 4.24$ & 2.03 & - \\
\hline GalNAc4,6S( $(\beta)$ & $(100.4)$ & $(52.0)$ & $(76.7)$ & $(76.5)$ & $(72.4)$ & $(67.7)$ & $(22.9)$ & $(173.3)$ \\
\hline $\mathbf{T}$ & $3.69 / 3.80$ & 4.14 & 4.53 & 5.04 & 4.51 & $4.34 / 4.18$ & - & - \\
\hline anTal-ol4,6S & $(61.4)$ & $(81.4)$ & $(76.8)$ & $(77.8)$ & $(77.8)$ & $(68.5)$ & & \\
\hline \multicolumn{9}{|c|}{ FCS-Pg 12-mer $(n=2)$} \\
\hline $\mathbf{F}$ & 5.32 & 3.92 & 4.48 & 4.93 & 4.73 & 1.37 & - & - \\
\hline Fuc3,4S $(\alpha)$ & $(99.8)$ & $(66.9)$ & $(75.9)$ & $(80.0)$ & $(67.2)$ & $(16.5)$ & & \\
\hline $\mathrm{F}^{\prime}$ & 5.34 & 3.95 & 4.59 & 4.86 & 4.49 & 1.22 & - & - \\
\hline Fuc3,4S $(\alpha)$ & $(99.4)$ & $(66.9)$ & $(75.7)$ & $(79.6)$ & $(66.9)$ & $(16.3)$ & & \\
\hline U' & 4.56 & 3.58 & 3.62 & 3.73 & 3.90 & - & - & - \\
\hline $\operatorname{Glc} A(\beta)$ & $(104.0)$ & $(73.4)$ & $(81.3)$ & $(70.0)$ & $(75.5)$ & & & $(175.5)$ \\
\hline U & 4.52 & 3.62 & 3.76 & 4.11 & 3.93 & - & - & - \\
\hline $\operatorname{GlcA}(\beta)$ & $(104.0)$ & $(73.7)$ & $(79.7)$ & $(75.4)$ & $(75.5)$ & & & $(173.3)$ \\
\hline $\mathbf{U}$ & 4.59 & 3.71 & 3.74 & 4.08 & 4.01 & - & - & - \\
\hline $\operatorname{Glc} A(\beta)$ & (101.9) & $(73.5)$ & $(79.6)$ & $(75.4)$ & $(75.7)$ & & & $(173.3)$ \\
\hline $\mathbf{A}$ & 4.56 & 4.05 & 3.97 & 4.80 & 4.02 & $4.28 / 4.23$ & 2.03 & - \\
\hline GalNAc4,6S $(\beta)$ & $(100.4)$ & $(52.0)$ & $(76.7)$ & $(76.4)$ & $(72.3)$ & $(67.7)$ & $(22.8)$ & $(173.4)$ \\
\hline $\mathbf{T}$ & $3.69 / 3.78$ & 4.14 & 4.52 & 5.03 & 4.51 & $4.33 / 4.18$ & - & - \\
\hline anTal-ol4,6S & $(61.4)$ & $(81.3)$ & $(76.6)$ & $(77.8)$ & $(77.8)$ & $(68.4)$ & & \\
\hline \multicolumn{9}{|c|}{ FCS-Pg 15-mer $(n=3)$} \\
\hline $\mathbf{F}$ & 5.32 & 3.92 & 4.47 & 4.94 & 4.78 & 1.36 & - & - \\
\hline Fuc $3,4 \mathrm{~S}(\alpha)$ & $(99.7)$ & $(66.9)$ & $(76.0)$ & $(80.0)$ & $(67.1)$ & (16.4) & & \\
\hline F' & 5.34 & 3.93 & 4.58 & 4.86 & 4.48 & 1.22 & - & - \\
\hline
\end{tabular}




\begin{tabular}{|c|c|c|c|c|c|c|c|c|}
\hline Fuc3,4S $(\alpha)$ & (99.6) & $(66.9)$ & $(75.8)$ & (79.6) & (66.9) & $(16.2)$ & & \\
\hline U' & 4.54 & 3.57 & 3.61 & 3.72 & 3.87 & - & - & - \\
\hline $\operatorname{Glc} A(\beta)$ & (104.1) & (73.6) & (81.4) & $(70.2)$ & $(75.8)$ & & & (175.5) \\
\hline $\mathbf{U}$ & 4.51 & 3.60 & 3.74 & 4.08 & 3.89 & - & - & - \\
\hline $\operatorname{Glc} A(\beta)$ & (104.2) & (73.9) & $(79.8)$ & $(75.6)$ & $(75.8)$ & & & (173.4) \\
\hline U', & 4.57 & 3.70 & 3.71 & 4.05 & 3.99 & - & - & - \\
\hline $\operatorname{GlcA}(\beta)$ & (101.9) & $(73.6)$ & $(79.6)$ & $(75.3)$ & $(75.5)$ & & & (173.2) \\
\hline A & 4.53 & 4.05 & 3.96 & 4.78 & 4.01 & $4.28 / 4.23$ & 2.03 & - \\
\hline GalNAc4,6S( $\beta)$ & $(100.3)$ & $(52.0)$ & (76.6) & (76.6) & (72.4) & $(67.8)$ & $(23.0)$ & $(173.5)$ \\
\hline T & $3.67 / 3.78$ & 4.13 & 4.52 & 5.03 & 4.50 & $4.32 / 4.17$ & - & - \\
\hline \multicolumn{8}{|c|}{ FCS-Pg 18-mer $(n=4)$} & \\
\hline F & 5.32 & 3.92 & 4.46 & 4.94 & 4.77 & 1.36 & - & - \\
\hline Fuc $3,4 \mathrm{~S}(\alpha)$ & (99.9) & $(67.0)$ & $(76.0)$ & (79.9) & $(67.2)$ & $(16.5)$ & & \\
\hline F' & 5.34 & 3.94 & 4.57 & 4.85 & 4.47 & 1.22 & - & - \\
\hline Fuc $3,4 \mathrm{~S}(\alpha)$ & (99.7) & $(67.0)$ & $(75.8)$ & (79.7) & $(67.0)$ & $(16.3)$ & & \\
\hline U' & 4.54 & 3.57 & 3.61 & 3.72 & 3.88 & - & - & - \\
\hline $\operatorname{GlcA}(\beta)$ & (104.2) & $(74.0)$ & $(81.5)$ & $(70.2)$ & (75.7) & & & (175.4) \\
\hline $\mathbf{U}$ & 4.50 & 3.61 & 3.73 & 4.08 & 3.91 & - & - & - \\
\hline $\operatorname{GlcA}(\beta)$ & (104.4) & $(74.0)$ & (79.7) & (75.6) & (75.7) & & & (173.2) \\
\hline U', & 4.58 & 3.69 & 3.71 & 4.06 & 3.99 & - & - & - \\
\hline $\operatorname{Glc} A(\beta)$ & $(102.0)$ & $(73.7)$ & $(79.6)$ & $(75.3)$ & $(75.5)$ & & & $(173.1)$ \\
\hline $\mathbf{A}$ & 4.53 & 4.05 & 3.95 & 4.78 & 4.01 & $4.28 / 4.23$ & 2.02 & - \\
\hline GalNAc4,6S( $\beta)$ & $(100.5)$ & $(52.0)$ & $(76.9)$ & $(76.6)$ & $(72.4)$ & $(67.8)$ & $(23.0)$ & $(173.3)$ \\
\hline T & $3.67 / 3.78$ & 4.13 & 4.51 & 5.02 & 4.50 & $4.33 / 4.17$ & - & - \\
\hline anTal-ol4,6S & (61.4) & $(81.5)$ & $(76.8)$ & $(77.8)$ & (77.9) & $(68.5)$ & & \\
\hline
\end{tabular}



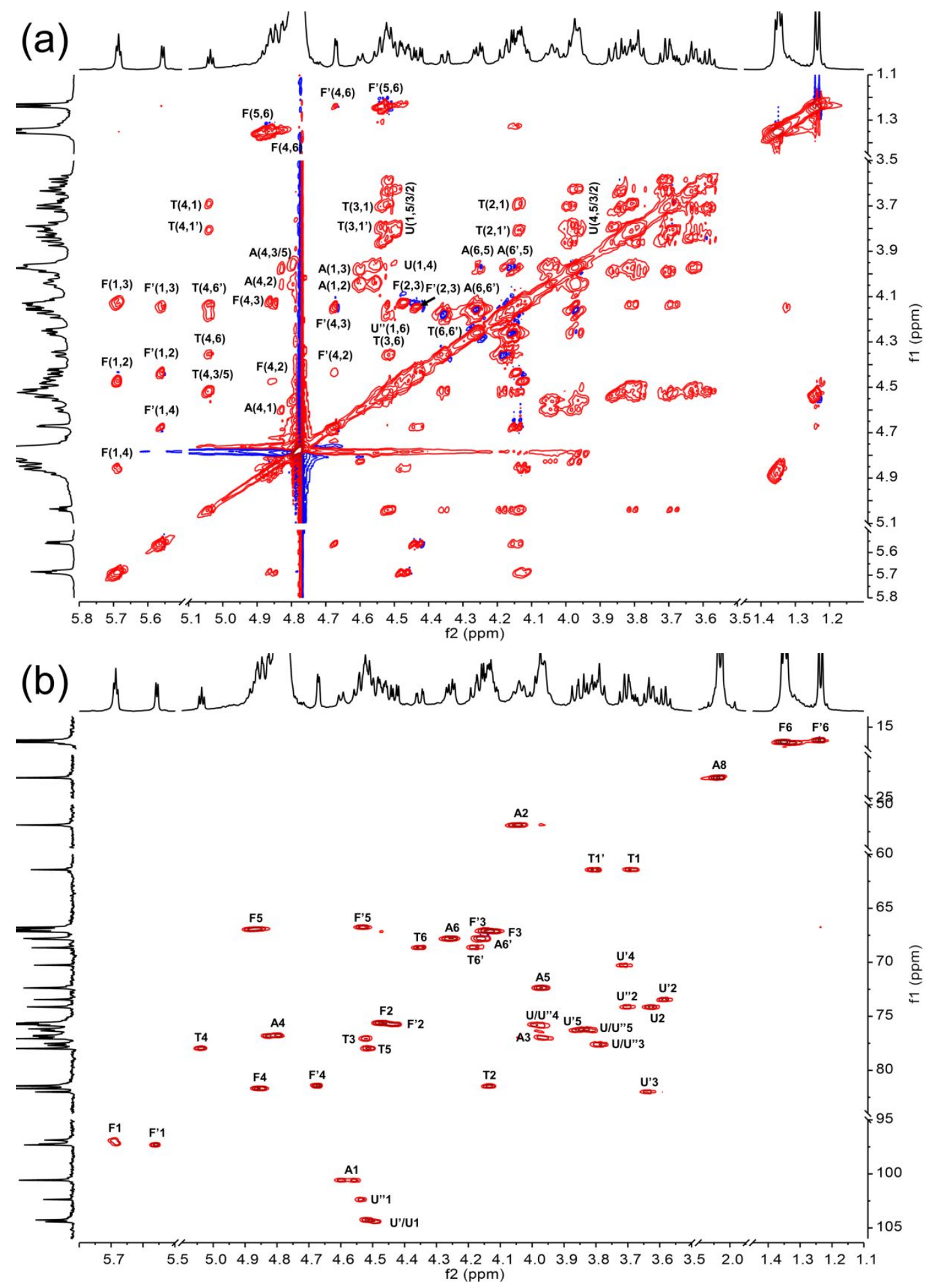

Figure S1. (a) ${ }^{1} \mathrm{H}-{ }^{1} \mathrm{H}$ TOCSY spectrum and (b) ${ }^{1} \mathrm{H}-{ }^{13} \mathrm{C}$ HSQC spectrum of FCS- $\mathrm{Ib}$ 9-mer. Labels are the same as those described in Table S1. 

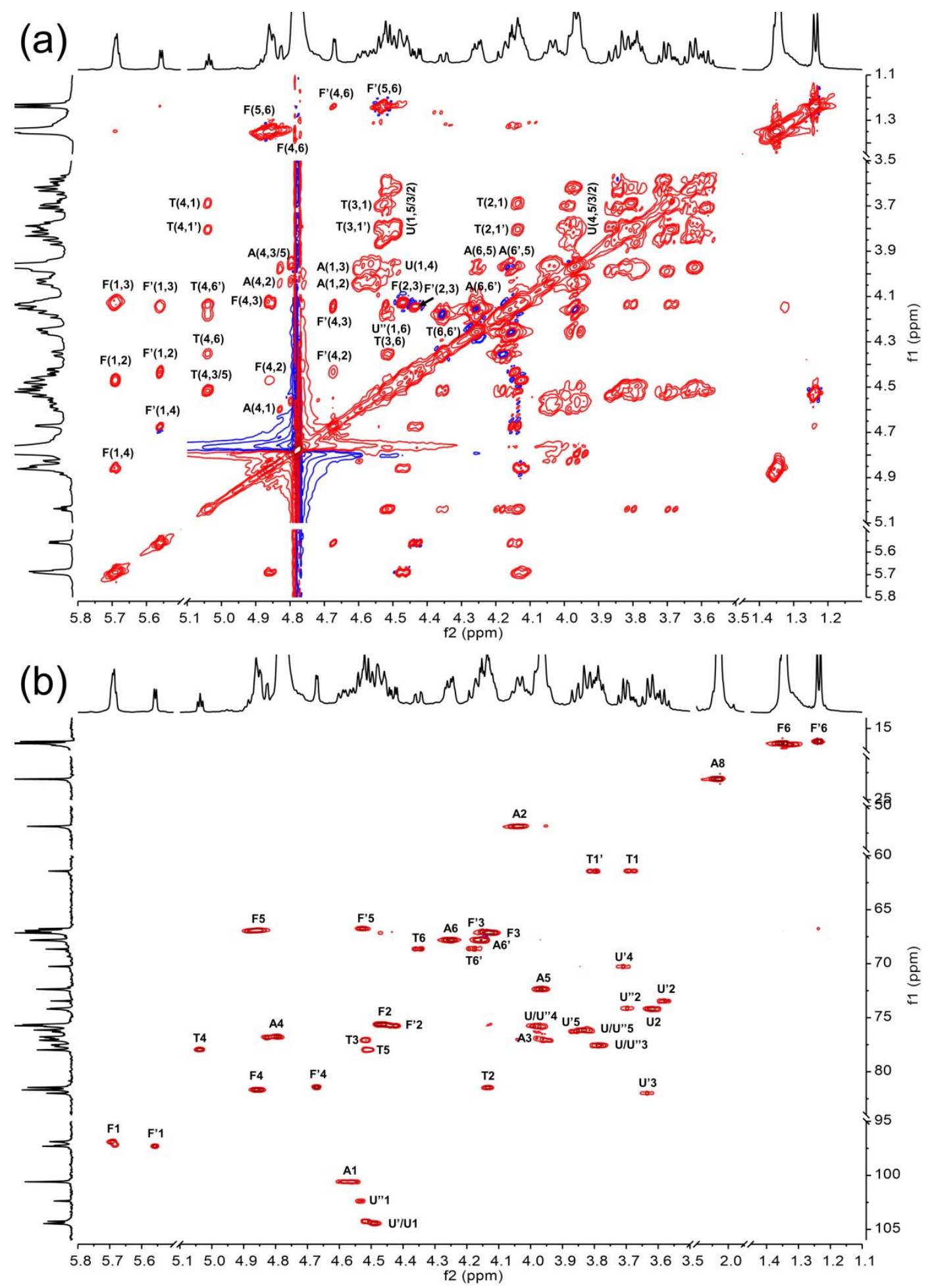

Figure S2. (a) ${ }^{1} \mathrm{H}-{ }^{1} \mathrm{H}$ TOCSY spectrum and (b) ${ }^{1} \mathrm{H}-{ }^{13} \mathrm{C}$ HSQC spectrum of FCS- $\mathrm{Ib}$ 12-mer. Labels are the same as those described in Table S1. 

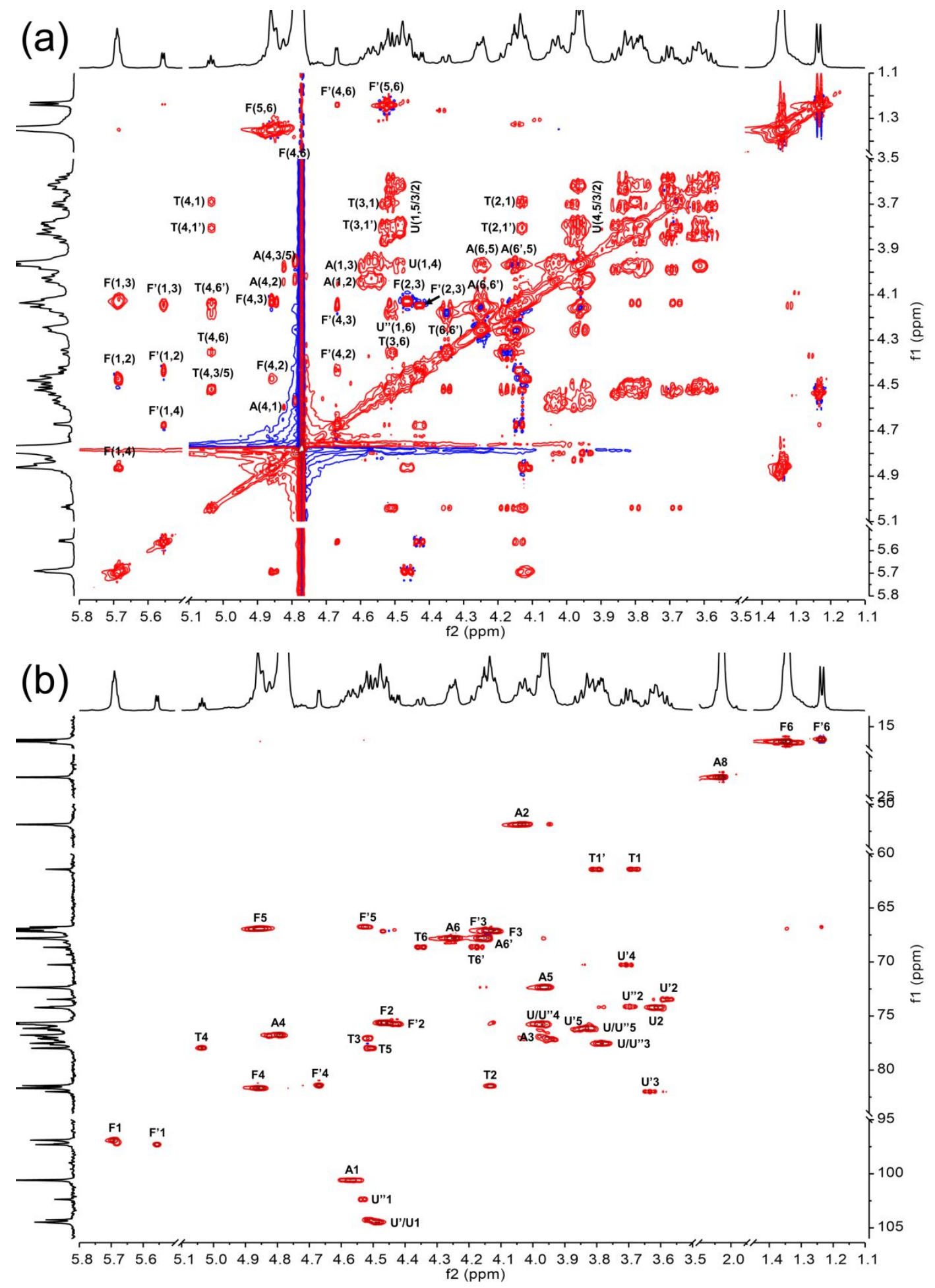

Figure S3. (a) ${ }^{1} \mathrm{H}-{ }^{1} \mathrm{H}$ TOCSY spectrum and (b) ${ }^{1} \mathrm{H}-{ }^{13} \mathrm{C}$ HSQC spectrum of FCS- $\mathrm{Ib}$ 15-mer. Labels are the same as those described in Table S1. 

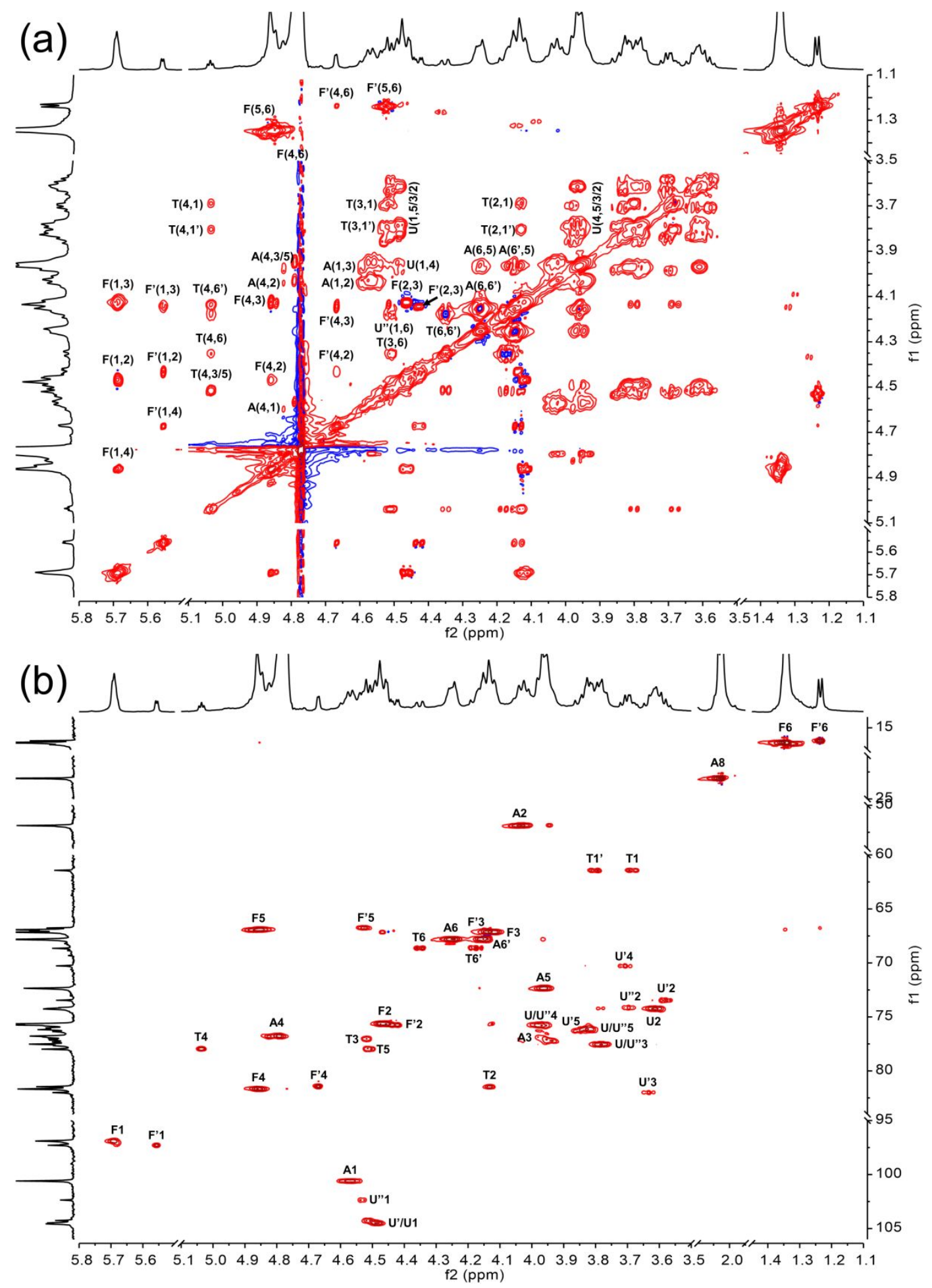

Figure S4. (a) ${ }^{1} \mathrm{H}-{ }^{1} \mathrm{H}$ TOCSY spectrum and (b) ${ }^{1} \mathrm{H}-{ }^{13} \mathrm{C}$ HSQC spectrum of FCS- $\mathrm{Ib}$ 18-mer. Labels are the same as those described in Table S1. 

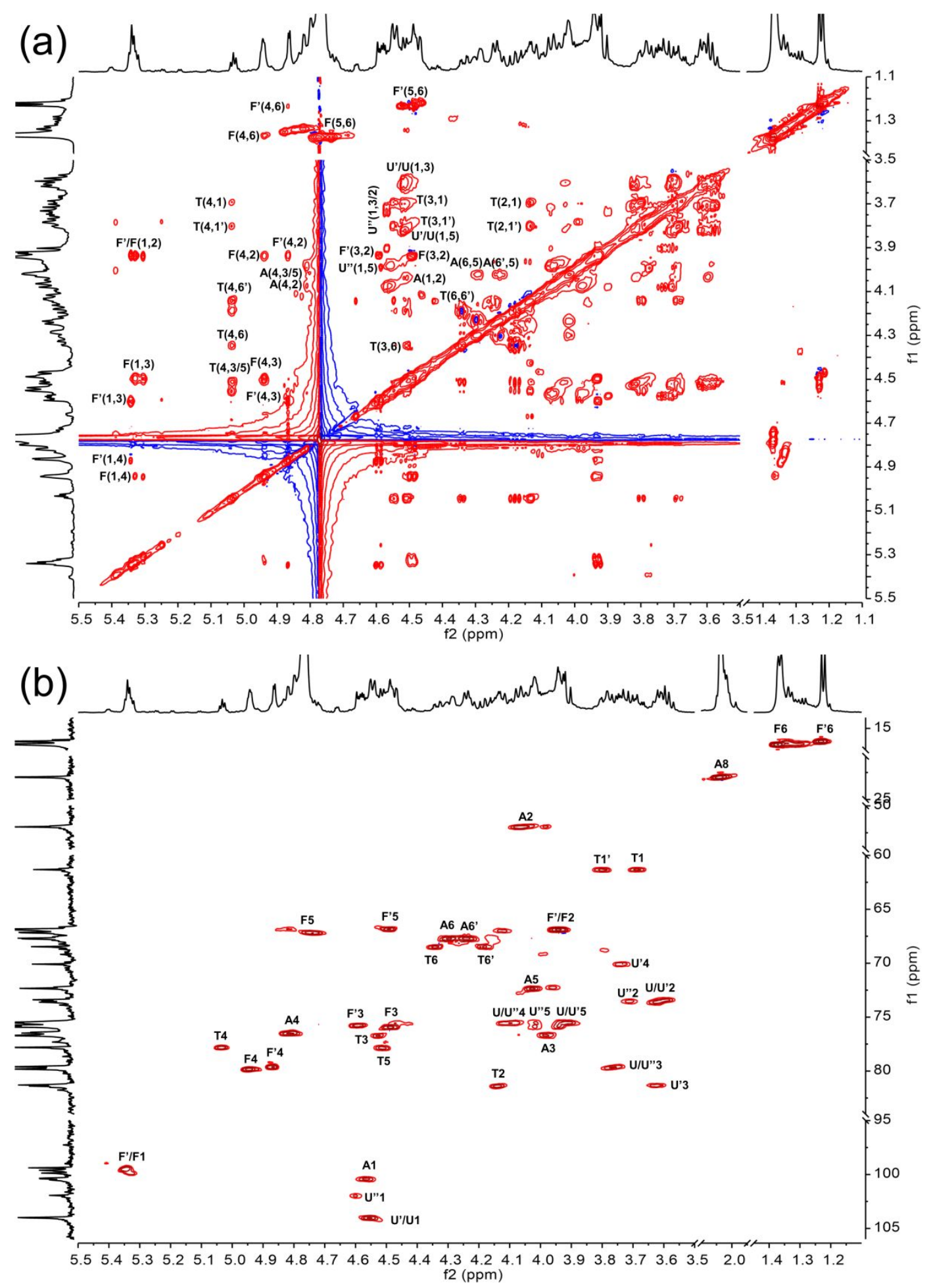

Figure S5. (a) ${ }^{1} \mathrm{H}-{ }^{1} \mathrm{H}$ TOCSY spectrum and (b) ${ }^{1} \mathrm{H}-{ }^{13} \mathrm{C}$ HSQC spectrum of FCS- $P g$ 9-mer. Labels are the same as those described in Table S2. 

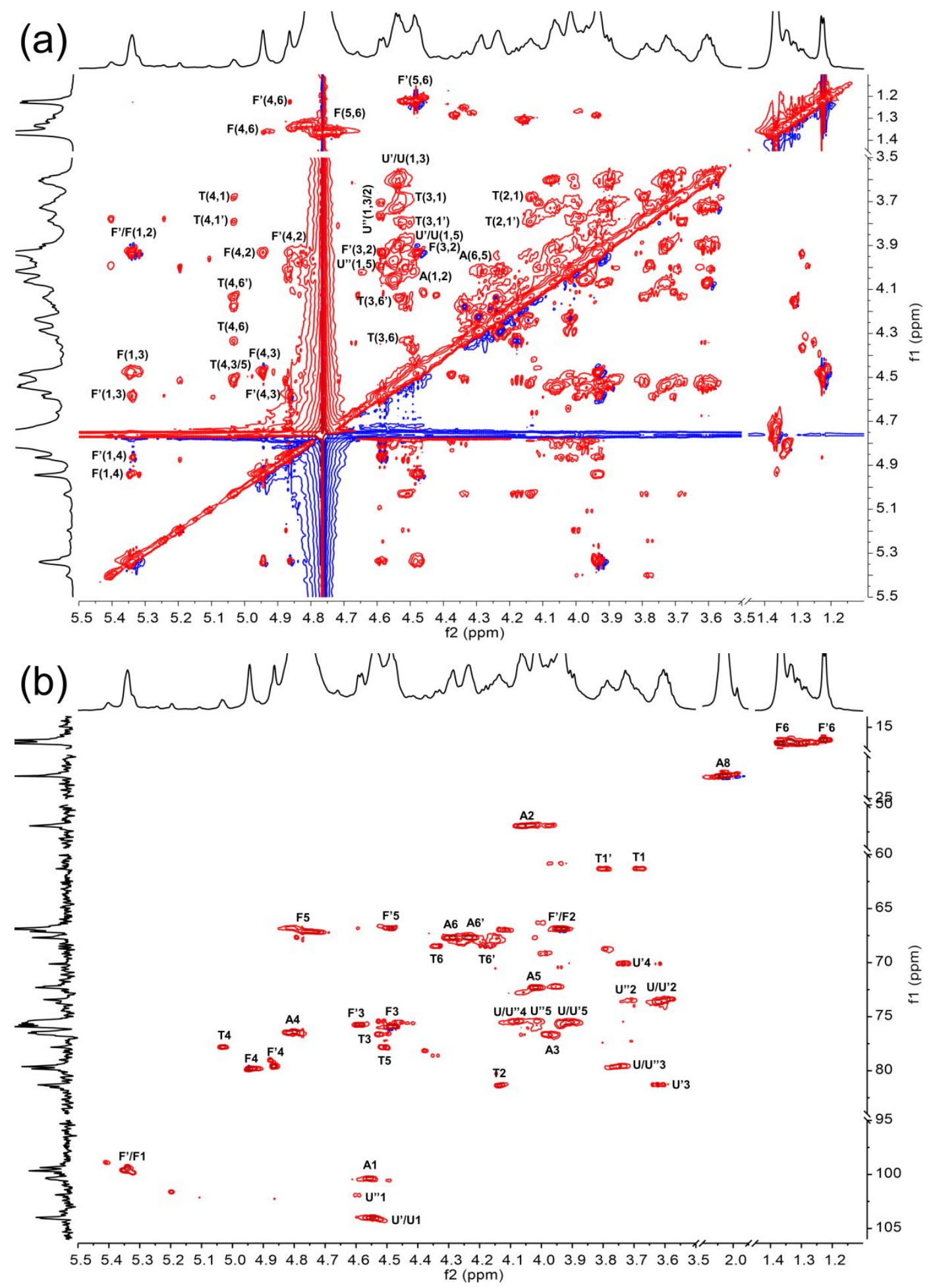

Figure S6. (a) ${ }^{1} \mathrm{H}-{ }^{1} \mathrm{H}$ TOCSY spectrum and (b) ${ }^{1} \mathrm{H}-{ }^{13} \mathrm{C}$ HSQC spectrum of FCS- $P g$ 12-mer. Labels are the same as those described in Table S2. 

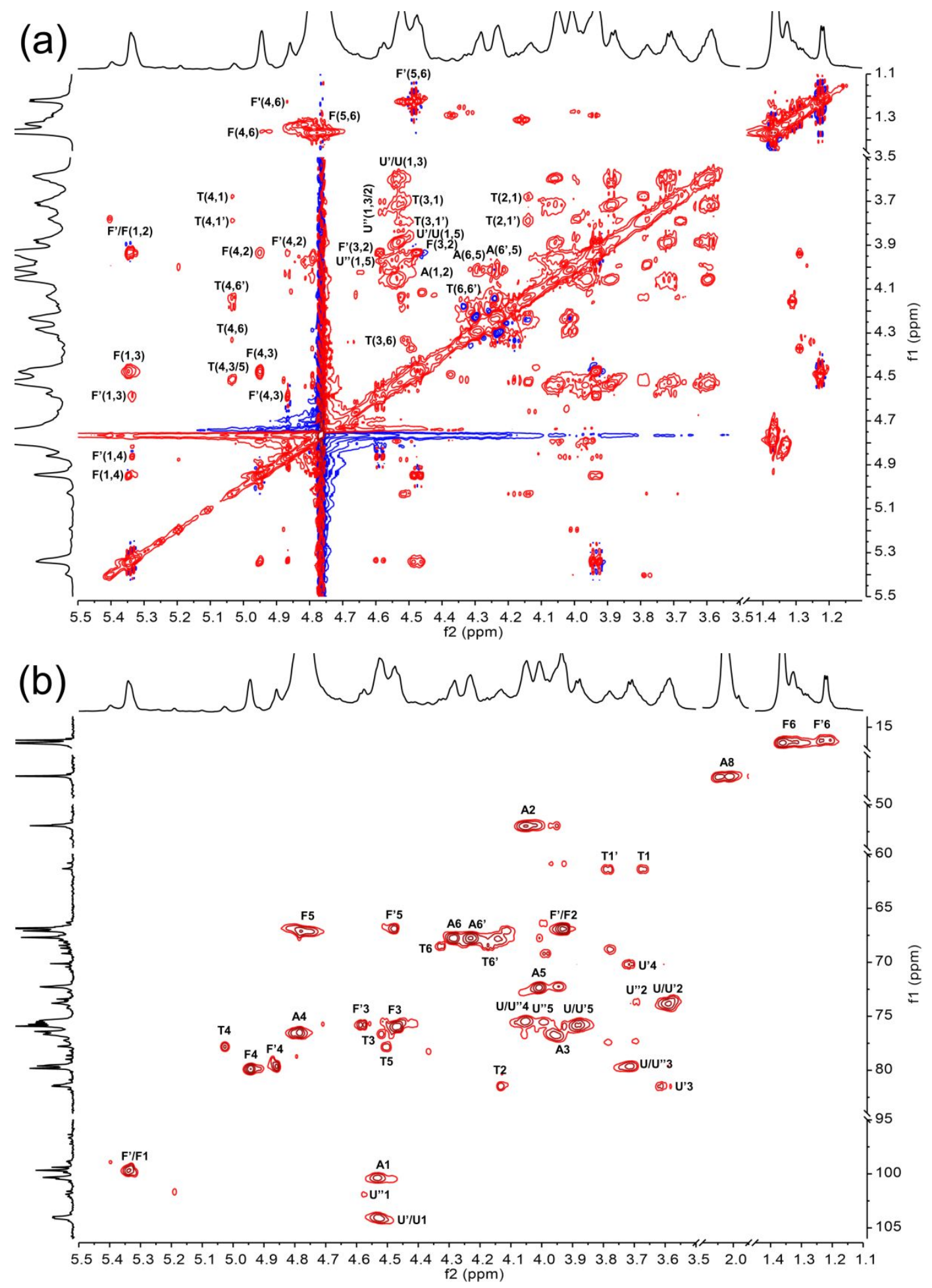

Figure S7. (a) ${ }^{1} \mathrm{H}-{ }^{1} \mathrm{H}$ TOCSY spectrum and (b) ${ }^{1} \mathrm{H}-{ }^{13} \mathrm{C}$ HSQC spectrum of FCS- $P g$ 15-mer. Labels are the same as those described in Table S2. 

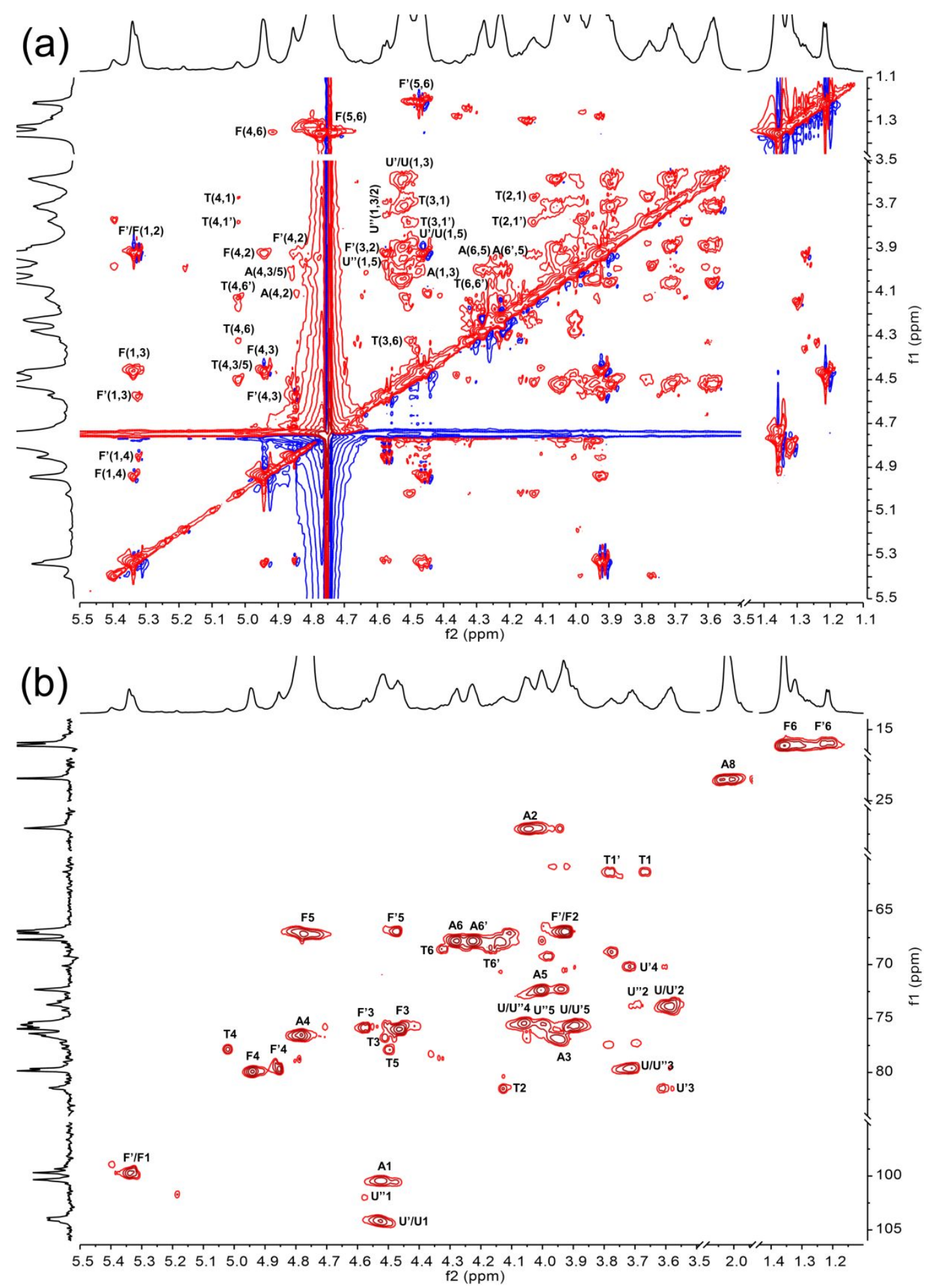

Figure S8. (a) ${ }^{1} \mathrm{H}-{ }^{1} \mathrm{H}$ TOCSY spectrum and (b) ${ }^{1} \mathrm{H}-{ }^{13} \mathrm{C}$ HSQC spectrum of FCS- $P g$ 18-mer. Labels are the same as those described in Table S2. 

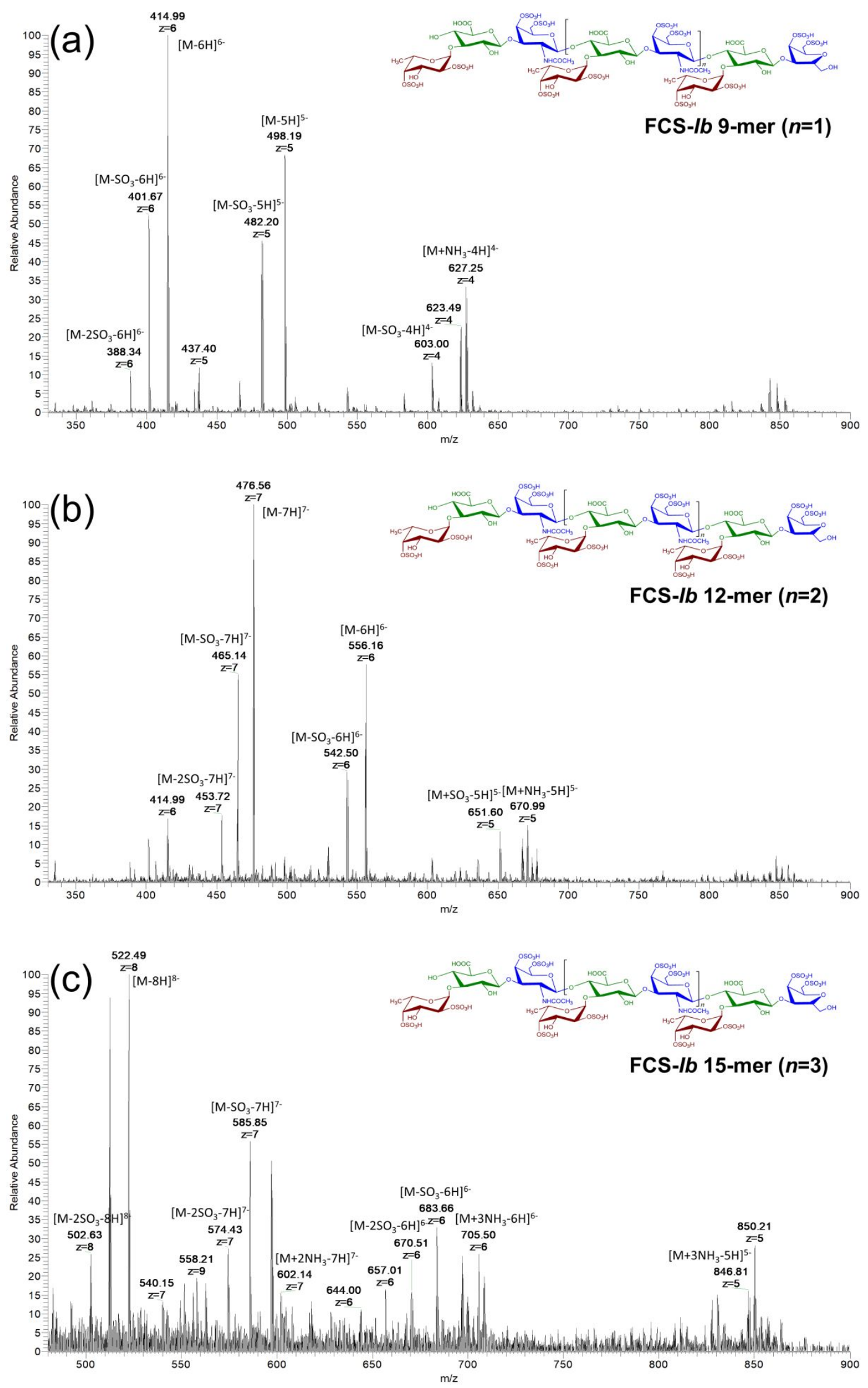


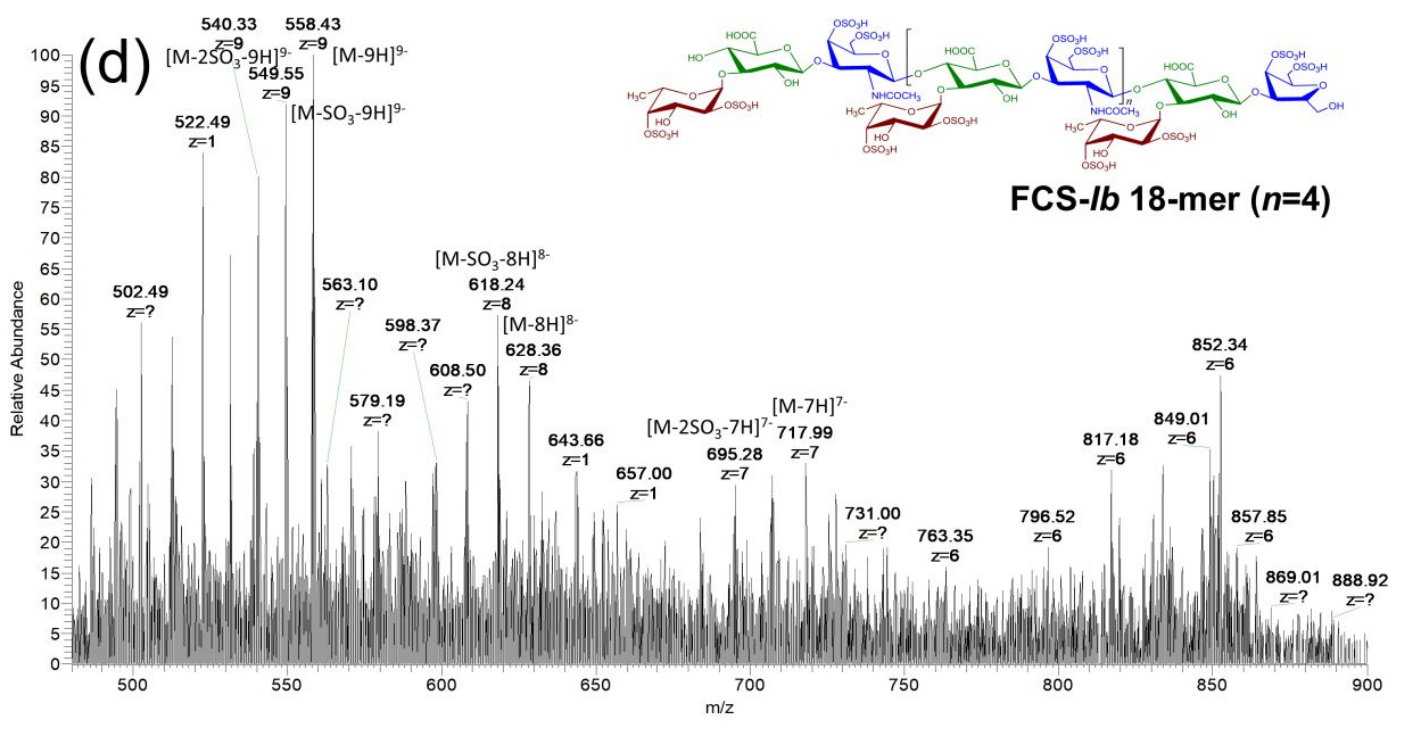

Figure S9. High-resolution FTMS spectrograms of (a) FCS-Ib 9-mer, (b) FCS-Ib 12-mer, (c) FCS-Ib 15-mer, (d) FCS-Ib 18-mer with matched structures. The patterns of sulfation in the fucose residues are based on NMR spectra analysis. 

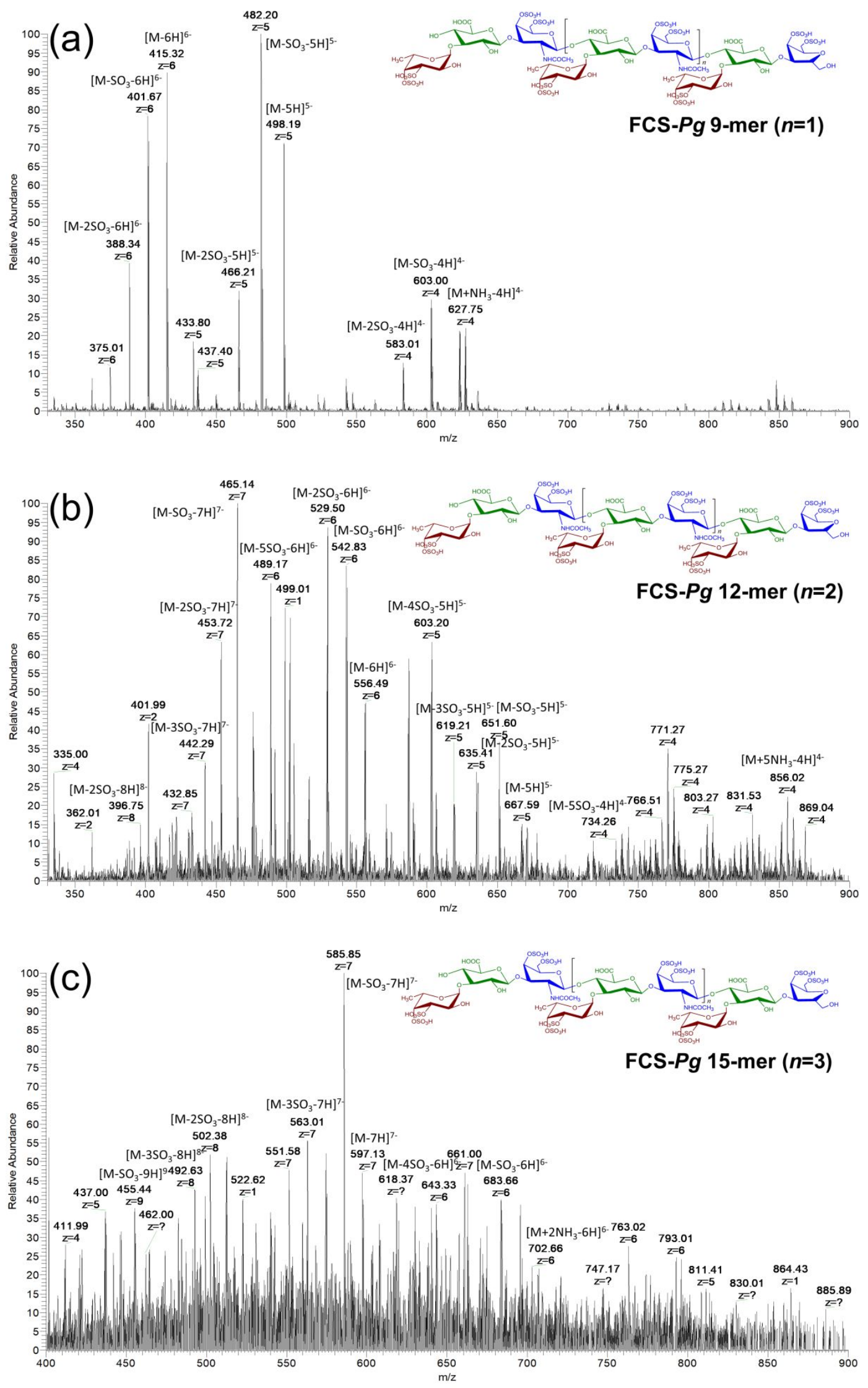


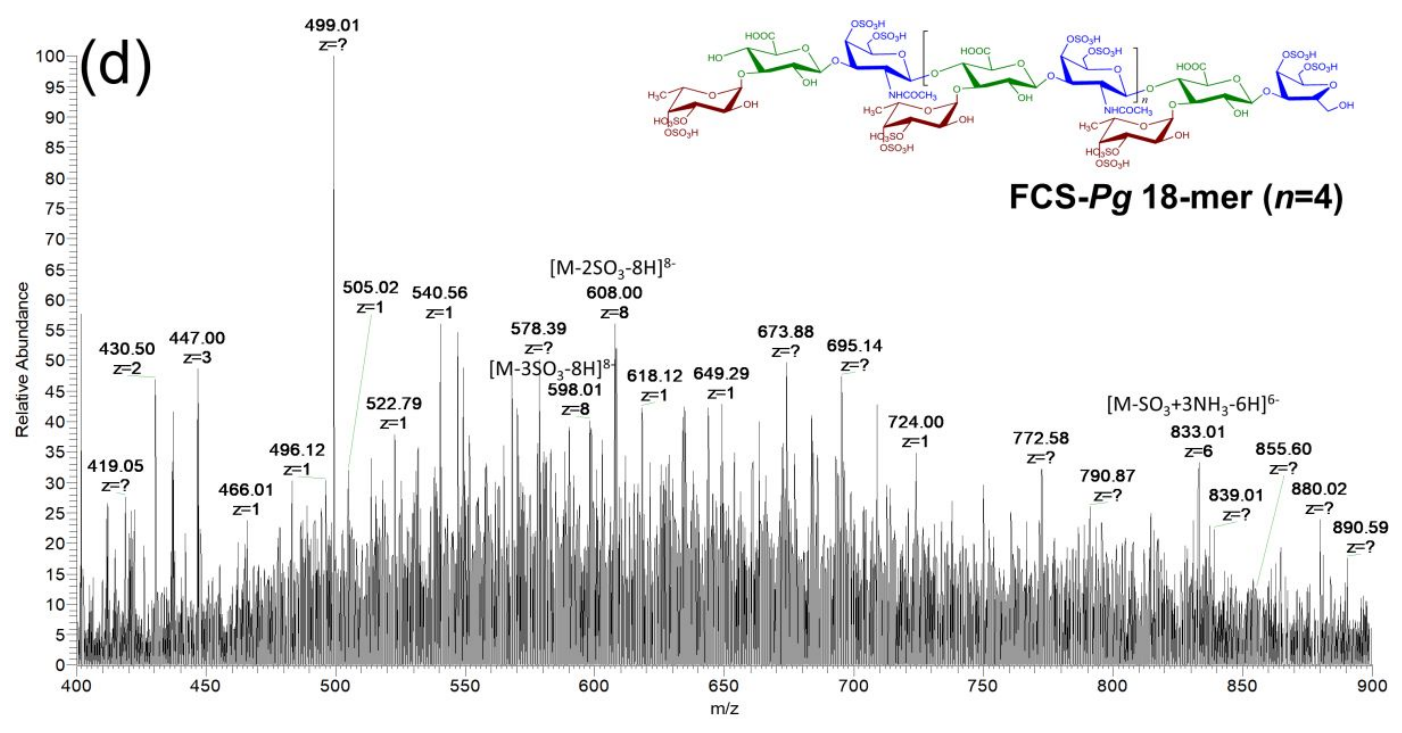

Figure S10. High-resolution FTMS spectrograms of (a) FCS- $P g$ 9-mer, (b) FCS- $P g$ 12-mer, (c) FCS-Pg 15-mer, (d) FCS-Pg 18-mer with matched structures. The patterns of sulfation in the fucose residues are based on NMR spectra analysis. 

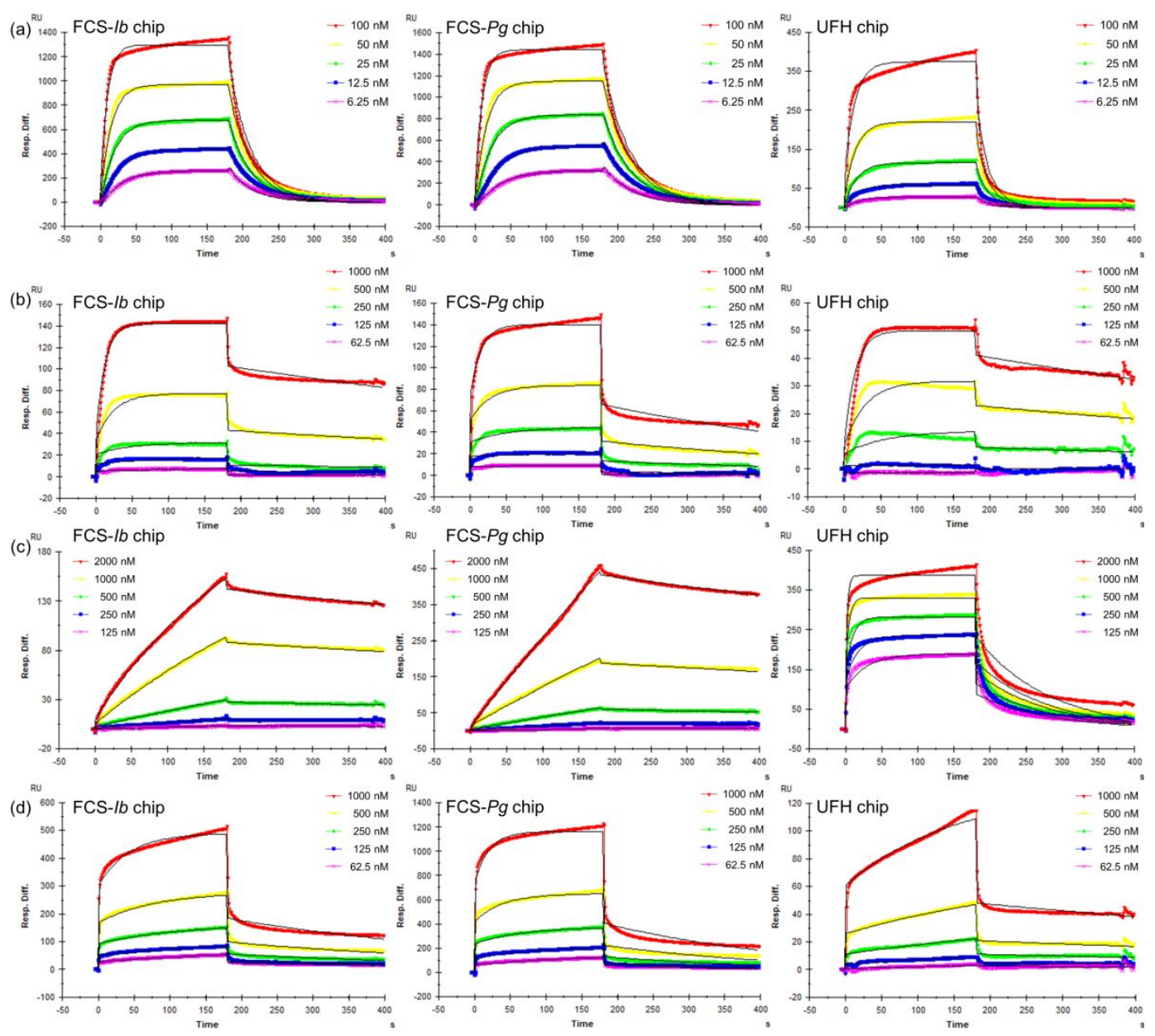

Figure S11. SPR sensorgrams with fitted curve. Gradient concentrations of (a) FIIa,

(b) FXa, (c) AT and (d) FIXa interact with immobilized FCS-Ib, FCS-Pg and UFH, respectively. 
Table S3 Kinetic constants of interactions between coagulation factors or cofactor and immobilized GAGs.

\begin{tabular}{lllll}
\hline Protein & Immobilized GAG & $k_{a}(1 / \mathrm{Ms})^{\mathrm{a}}$ & $k_{d}(1 / \mathrm{s})^{\mathrm{a}}$ & $K_{D}(\mathrm{M})$ \\
\hline FIIa & FCS- $\mathrm{Ib}$ & $7.22 \times 10^{5} \pm 1.48 \times 10^{4}$ & $3.13 \times 10^{-2} \pm 2.32 \times 10^{-4}$ & $4.33 \times 10^{-8}$ \\
& FCS- $P g$ & $7.44 \times 10^{5} \pm 1.24 \times 10^{4}$ & $2.54 \times 10^{-2} \pm 1.53 \times 10^{-4}$ & $3.42 \times 10^{-8}$ \\
& UFH & $1.54 \times 10^{5} \pm 2.05 \times 10^{4}$ & $5.69 \times 10^{-2} \pm 1.05 \times 10^{-3}$ & $3.70 \times 10^{-7}$ \\
FXa & FCS- $\mathrm{Ib}$ & $6.09 \times 10^{4} \pm 1.59 \times 10^{3}$ & $2.00 \times 10^{-3} \pm 3.36 \times 10^{-5}$ & $3.28 \times 10^{-8}$ \\
& FCS- $P g$ & $6.70 \times 10^{4} \pm 2.44 \times 10^{3}$ & $2.16 \times 10^{-3} \pm 7.13 \times 10^{-5}$ & $3.22 \times 10^{-8}$ \\
& UFH & $6.67 \times 10^{4} \pm 1.73 \times 10^{3}$ & $1.08 \times 10^{-3} \pm 4.63 \times 10^{-5}$ & $1.62 \times 10^{-8}$ \\
AT & FCS- $\mathrm{Ib}$ & $1.91 \times 10^{3} \pm 39.7$ & $5.25 \times 10^{-4} \pm 6.51 \times 10^{-6}$ & $2.76 \times 10^{-7}$ \\
& FCS- $P g$ & $556 \pm 15.2$ & $6.28 \times 10^{-4} \pm 5.73 \times 10^{-6}$ & $1.13 \times 10^{-6}$ \\
& UFH & $1.90 \times 10^{5} \pm 9.84 \times 10^{3}$ & $1.02 \times 10^{-2} \pm 1.68 \times 10^{-4}$ & $5.39 \times 10^{-8}$ \\
FIXa & FCS- $\mathrm{Ib}$ & $2.10 \times 10^{4} \pm 958$ & $2.39 \times 10^{-3} \pm 6.95 \times 10^{-5}$ & $1.14 \times 10^{-7}$ \\
& FCS- $P g$ & $4.44 \times 10^{4} \pm 2.38 \times 10^{3}$ & $3.66 \times 10^{-3} \pm 1.15 \times 10^{-4}$ & $8.22 \times 10^{-8}$ \\
& UFH & $5.54 \times 10^{3} \pm 582$ & $1.02 \times 10^{-3} \pm 5.16 \times 10^{-5}$ & $1.84 \times 10^{-7}$ \\
\hline
\end{tabular}

a The SD was from the global fitting. 
(a)

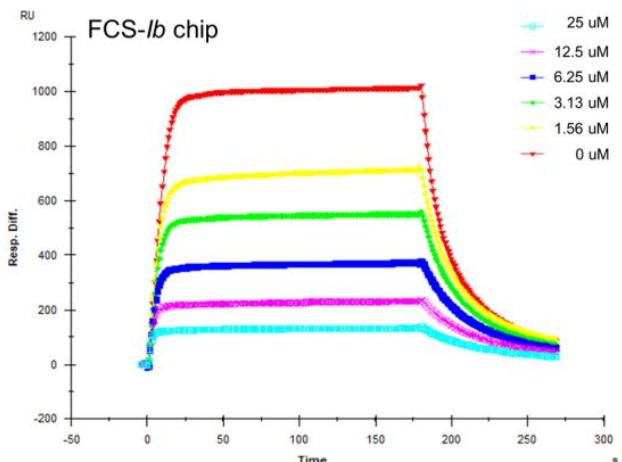

(b)

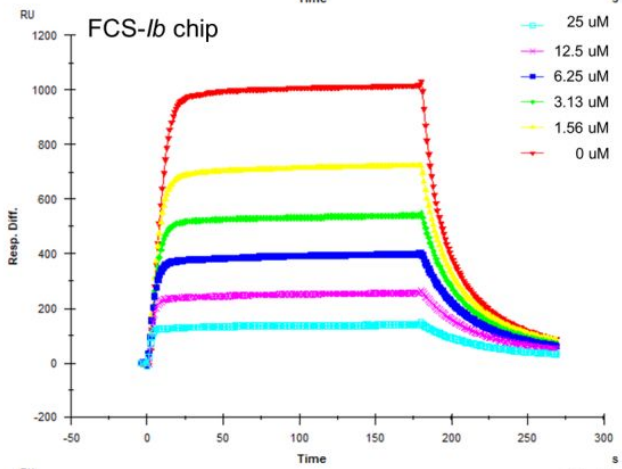

(c)

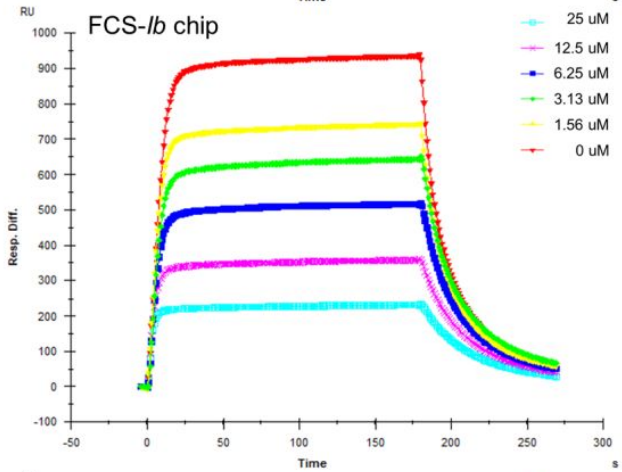

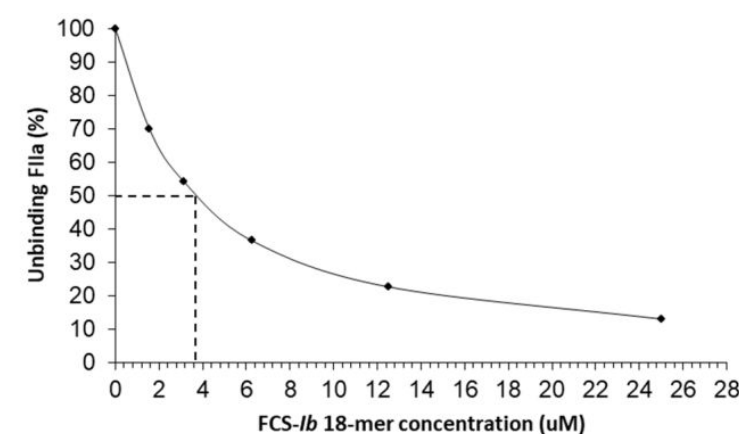
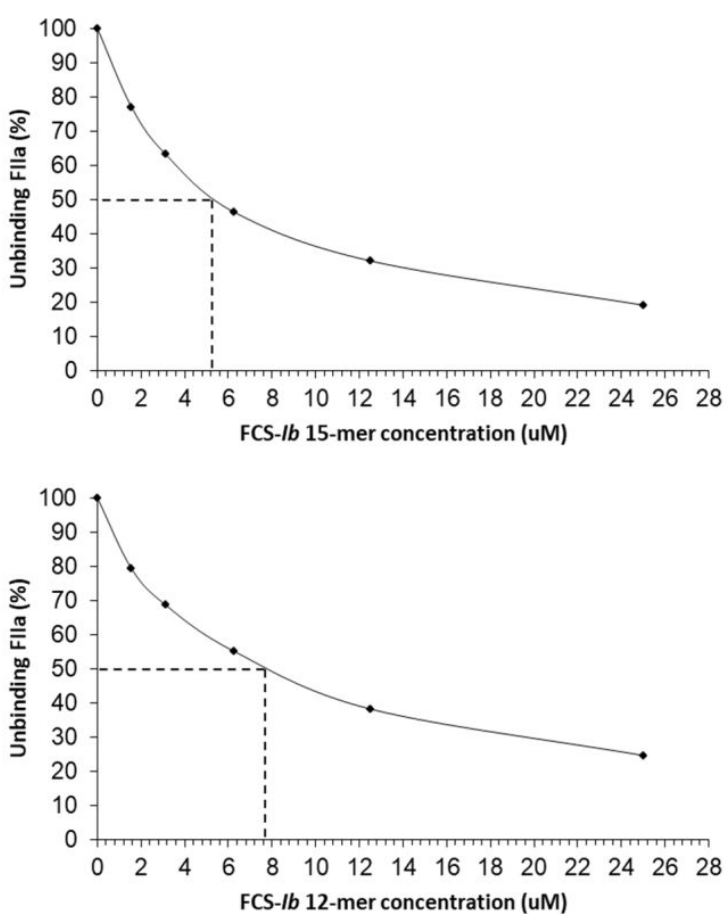

(d)

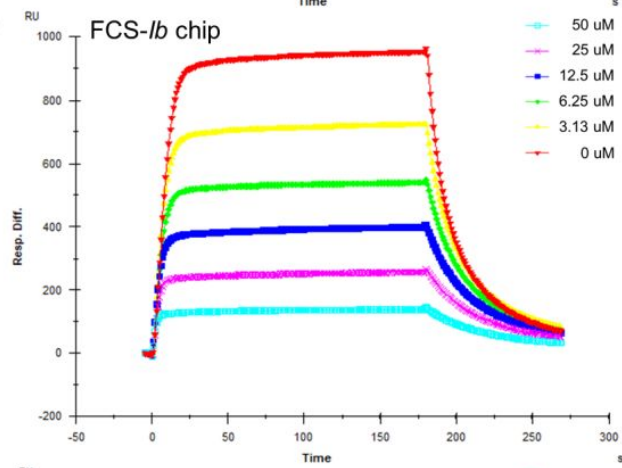

(e)

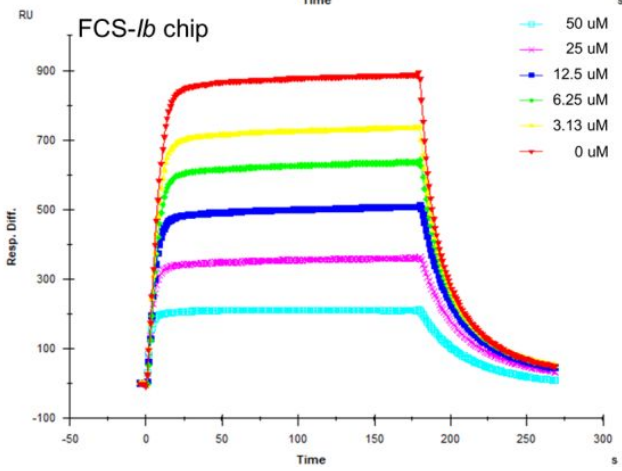

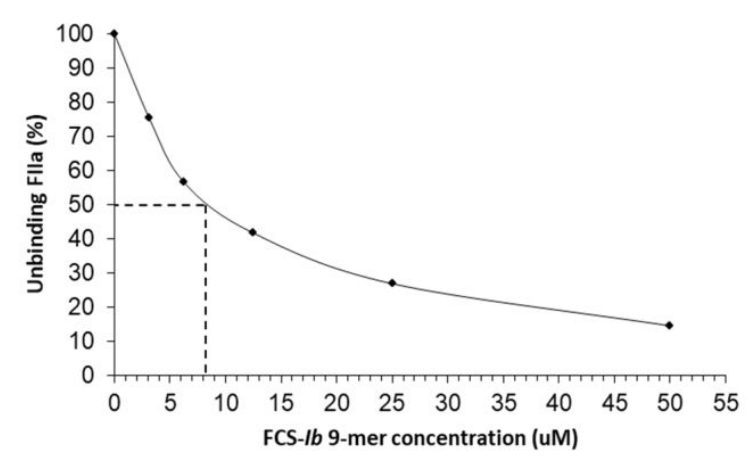

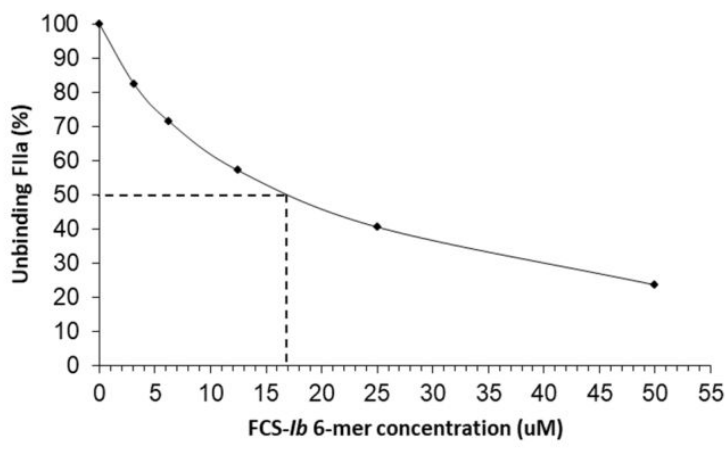



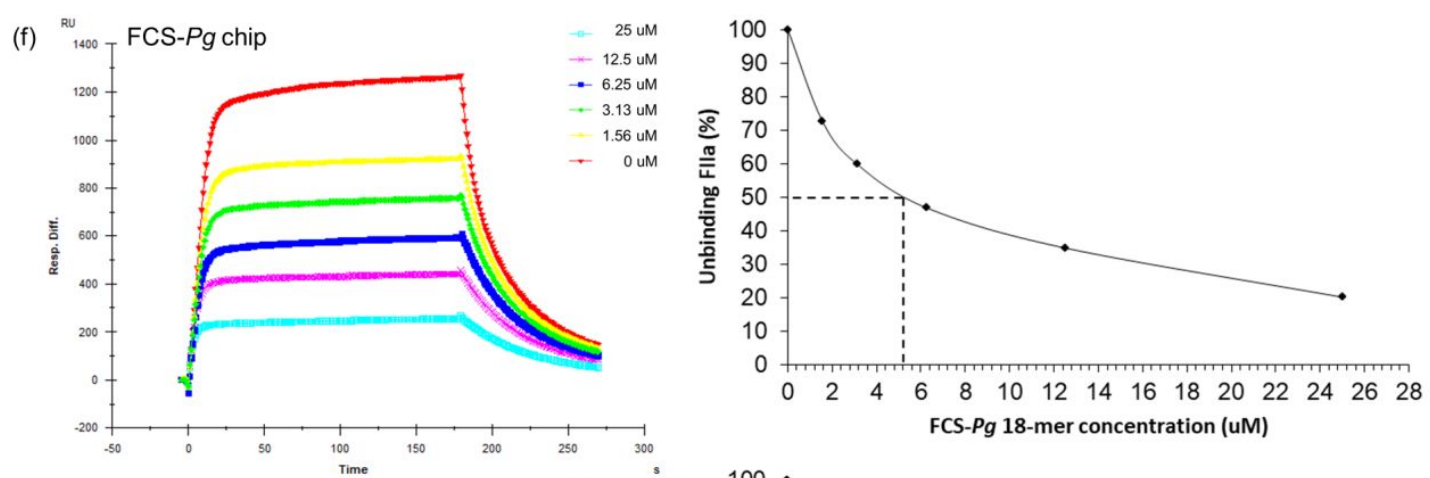

(g)
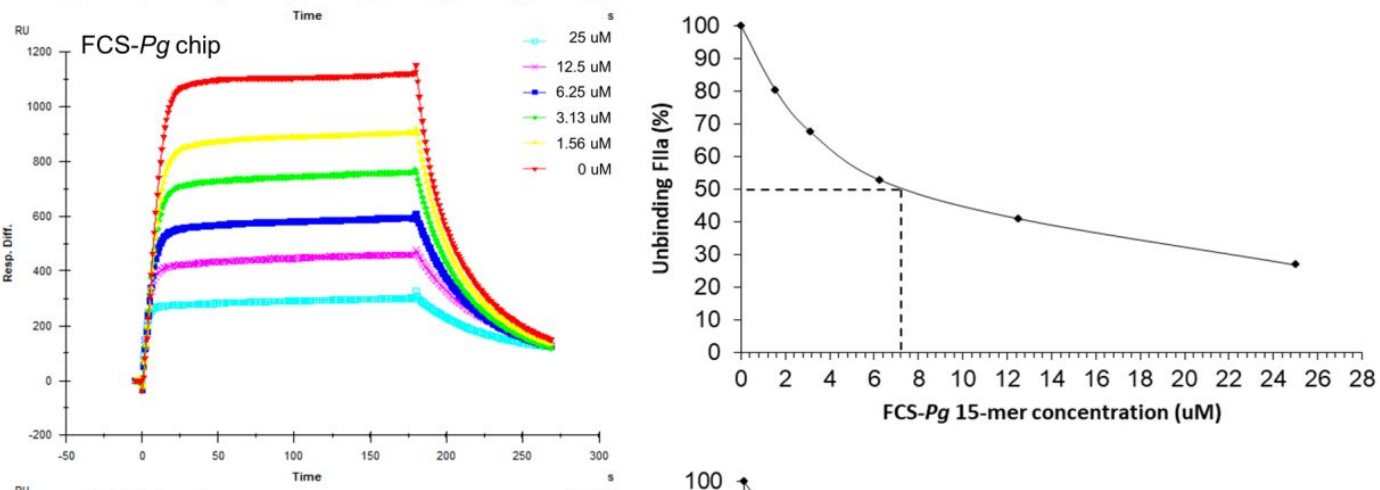

(h)
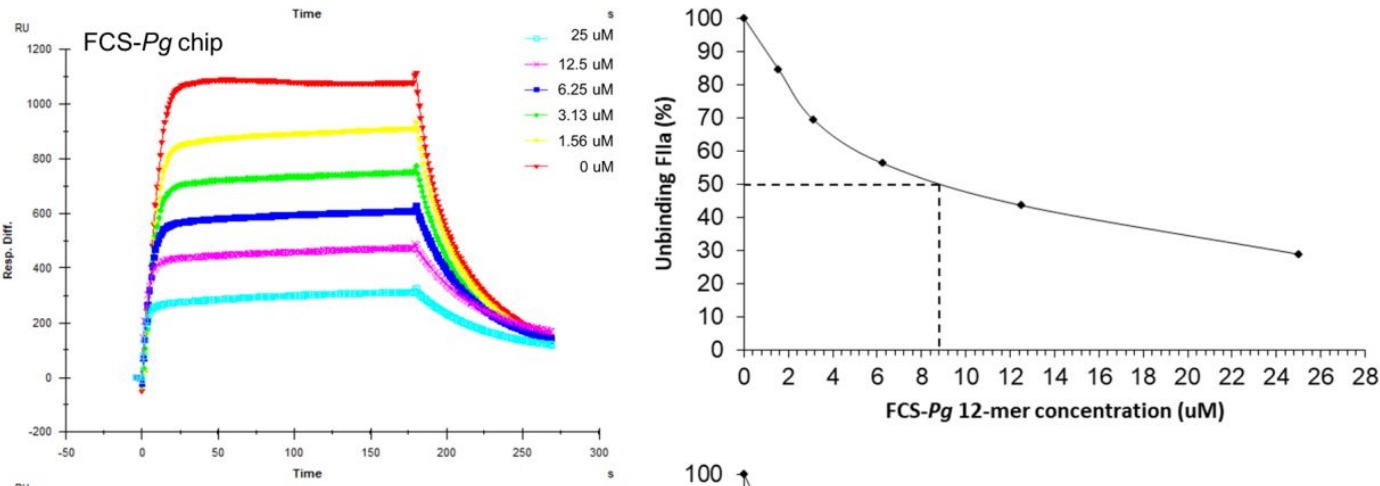

(i)
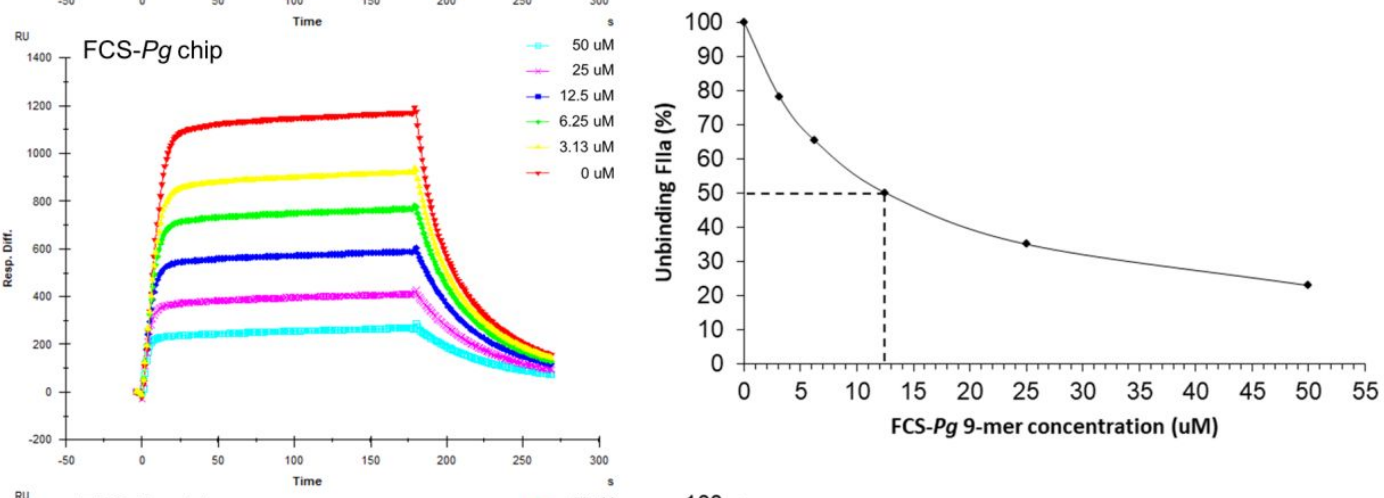

(j)
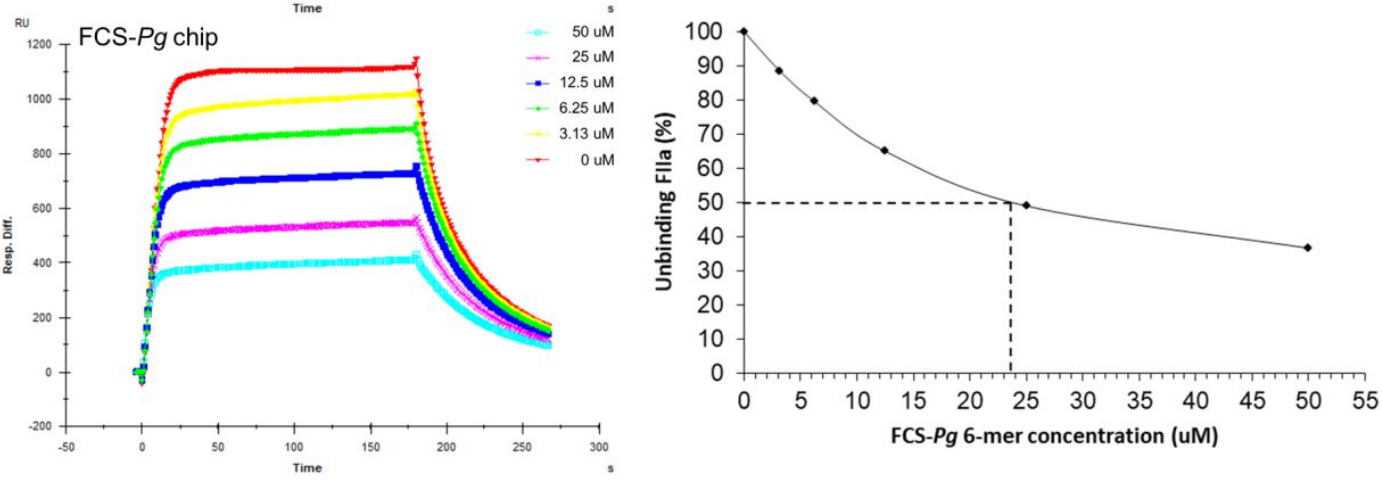

Figure S12. Competition of FIIa $(100 \mathrm{nM})$ binding to immobilized FCS-Ib by 
gradient concentrations of (a) FCS-Ib 18-mer, (b) FCS-Ib 15-mer, (c) FCS-Ib 12-mer, (d) FCS-Ib 9-mer and (e) FCS-Ib 6-mer with corresponding $\mathrm{IC}_{50}$. Competition of FIIa $(100 \mathrm{nM})$ binding to immobilized FCS- $P g$ by gradient concentrations of (f) FCS- $P g$ 18-mer, (g) FCS-Pg 15-mer, (h) FCS-Pg 12-mer, (i) FCS-Pg 9-mer and (j) FCS-Pg 6-mer with corresponding $\mathrm{IC}_{50}$. FIIa was preincubated with gradient concentrations of FCS oligomer prior to interact with immobilized FCS. The unbinding FIIa was the one not inhibited by FCS oligomer during preincubation. Unbinding FIIa (\%) was calculated by the highest response unit (RU) ratio of with and without preincubated FCS oligomer. 
(a)
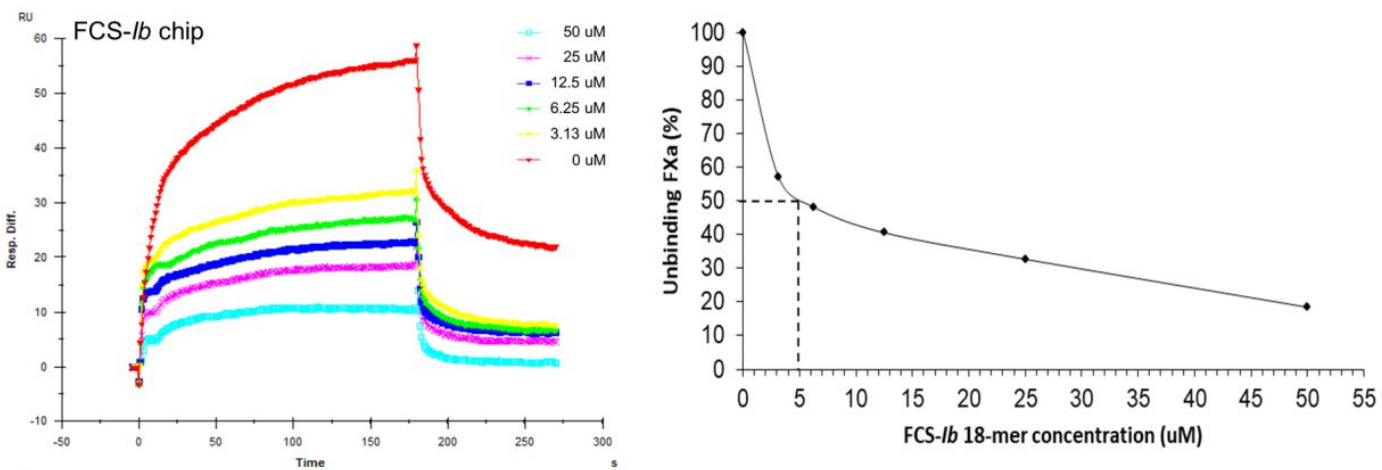

(b) ${ }_{60}^{\mathrm{RU}}$ FCS-Ib chip $\quad 50 \mathrm{um}$
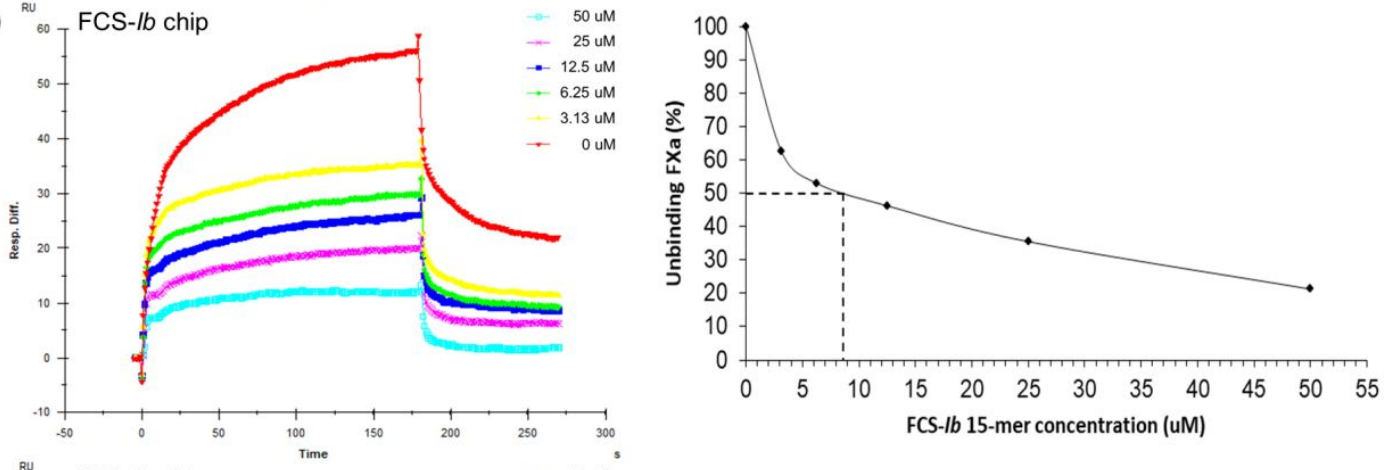

(c) ${ }_{60}^{\mathrm{RU}}$ FCS-Ib chip $\quad 50 \mathrm{um}$
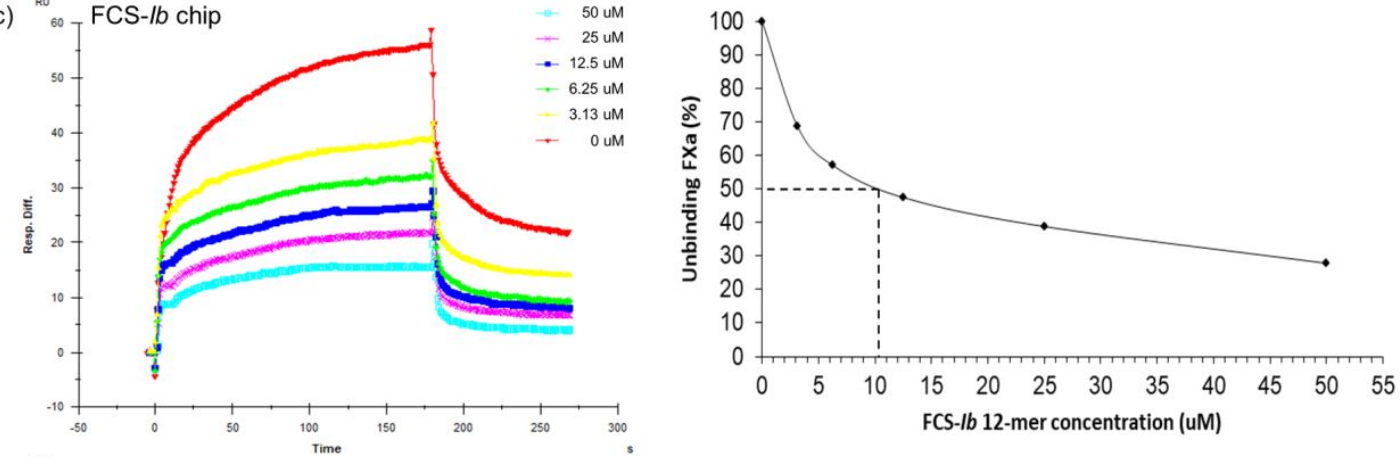

(d)
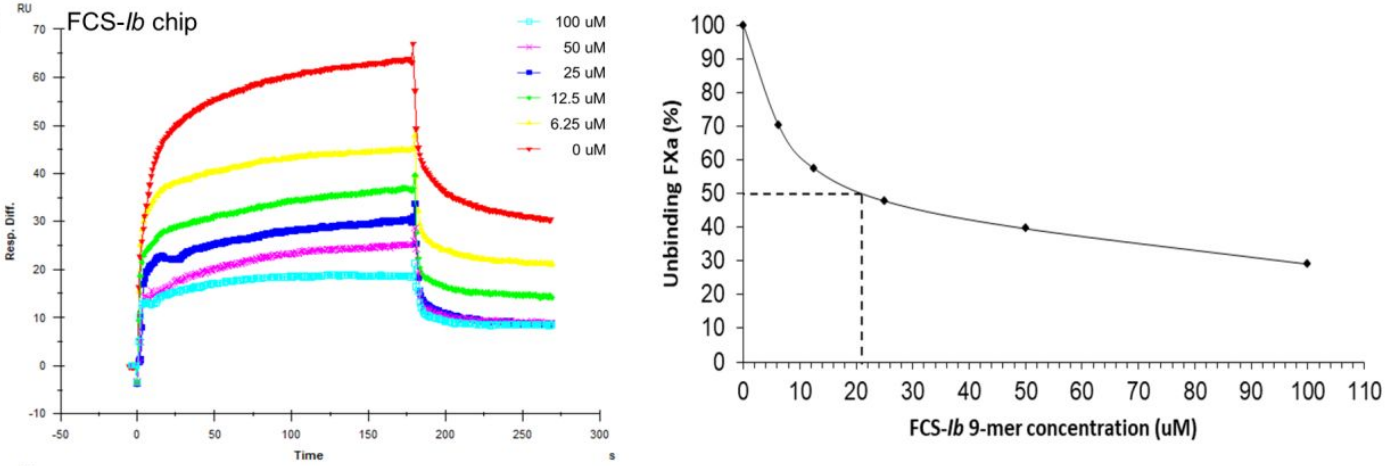

(e)
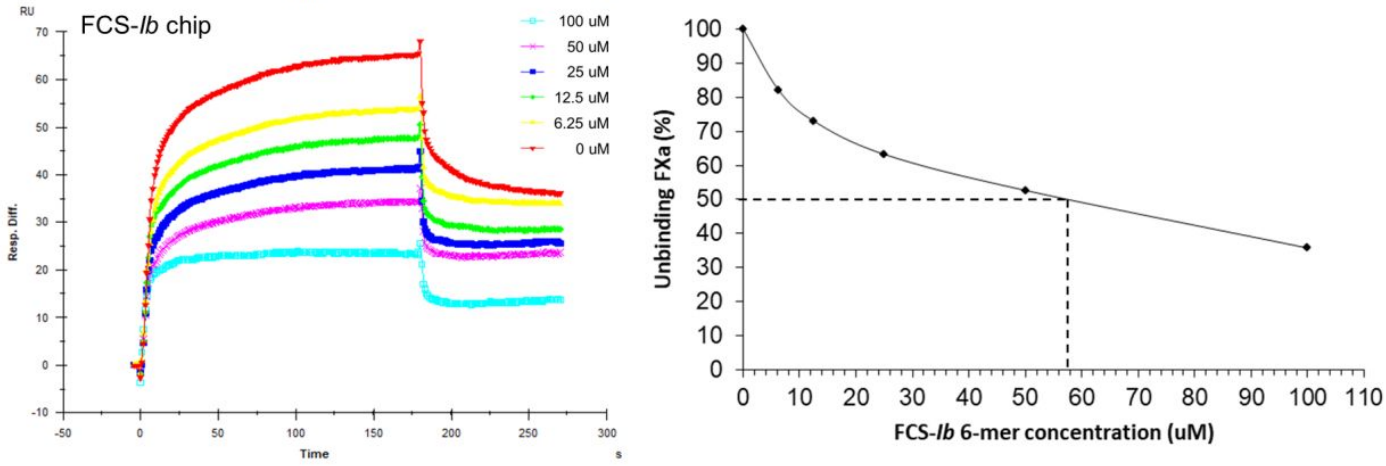
(f)

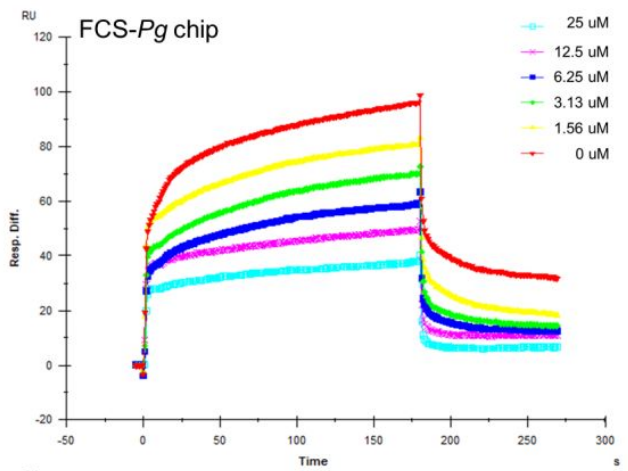

(g)

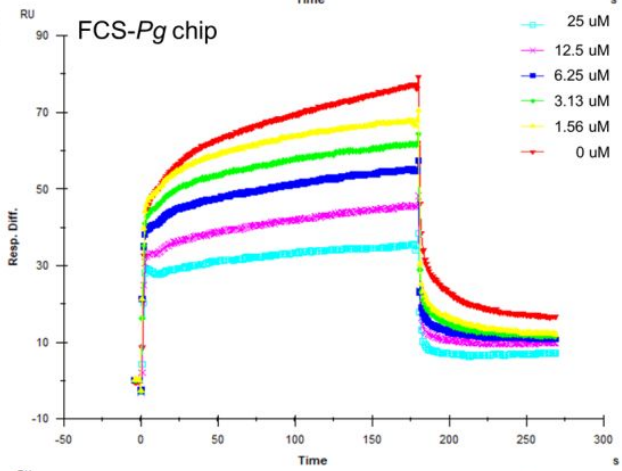

(h)

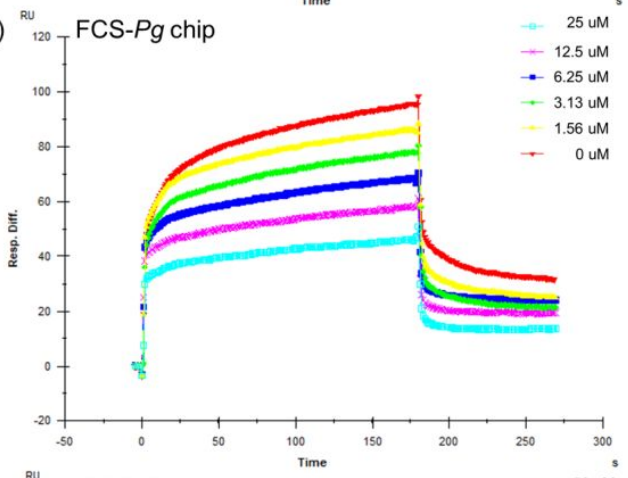

(i)

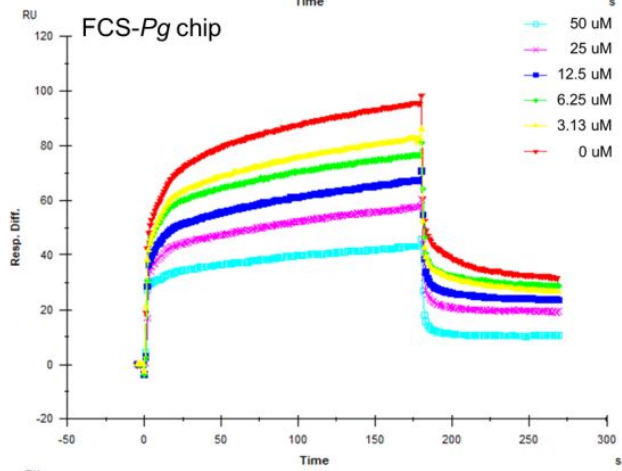

(j)

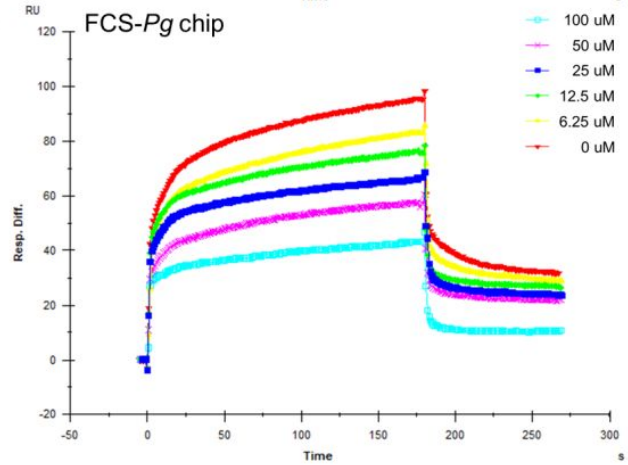

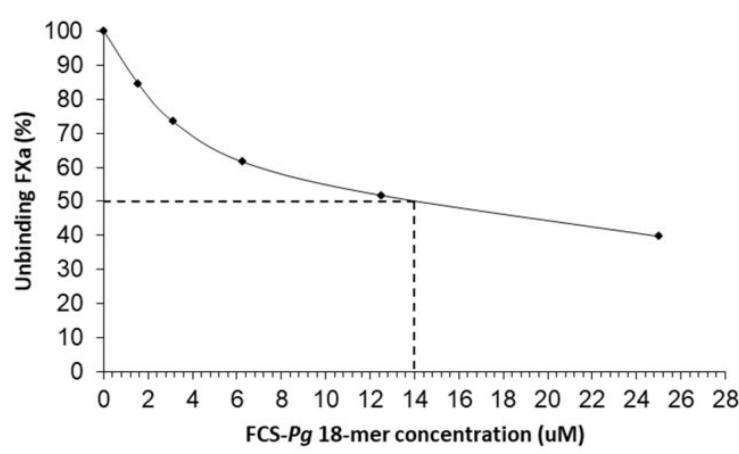
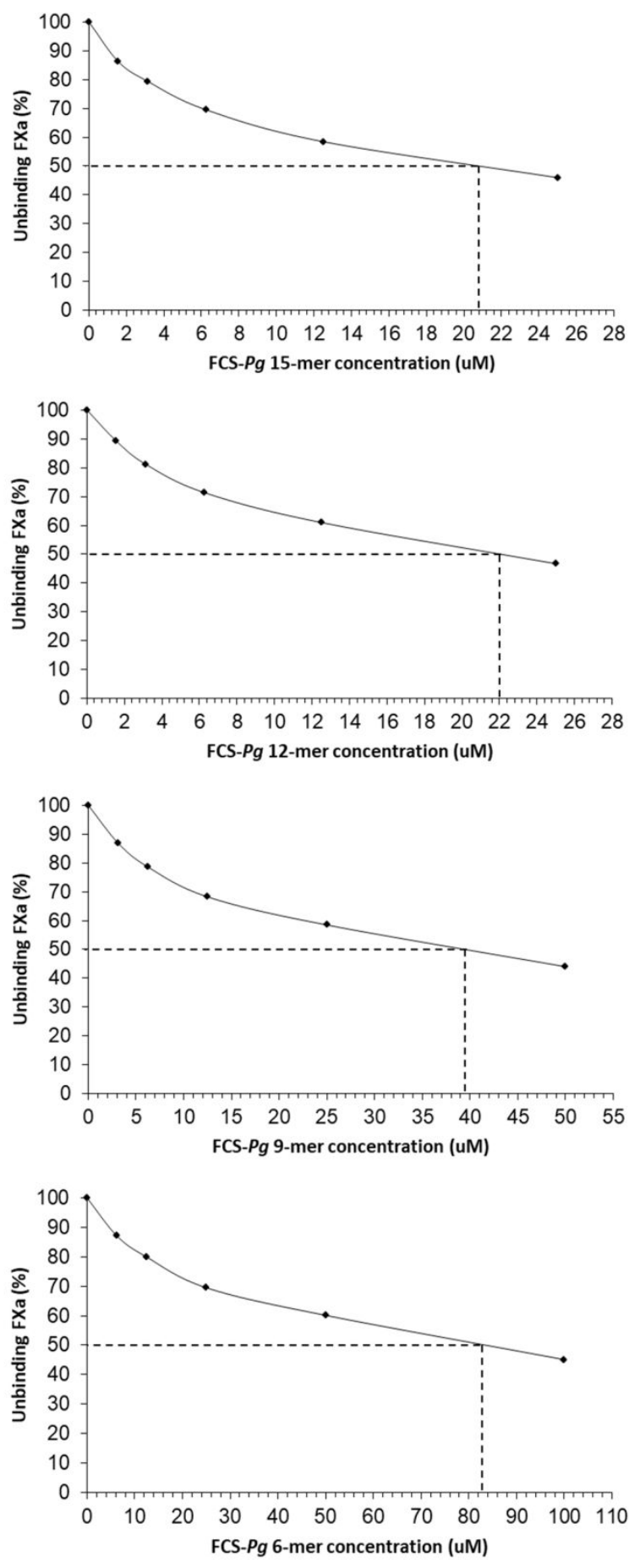

Figure S13. Competition of FXa $(500 \mathrm{nM})$ binding to immobilized FCS-Ib by 
gradient concentrations of (a) FCS-Ib 18-mer, (b) FCS-Ib 15-mer, (c) FCS-Ib 12-mer, (d) FCS-Ib 9-mer and (e) FCS-Ib 6-mer with corresponding IC $_{50}$. Competition of FXa (500 $\mathrm{nM}$ ) binding to immobilized FCS- $P g$ by gradient concentrations of (f) FCS- $P g$ 18-mer, (g) FCS-Pg 15-mer, (h) FCS-Pg 12-mer, (i) FCS-Pg 9-mer and (j) FCS-Pg 6-mer with corresponding $\mathrm{IC}_{50}$. FXa was preincubated with gradient concentrations of FCS oligomer prior to interact with immobilized FCS. The unbinding FXa was the one not inhibited by FCS oligomer during preincubation. Unbinding FXa (\%) was calculated by the highest response unit (RU) ratio of with and without preincubated FCS oligomer. 
(a)

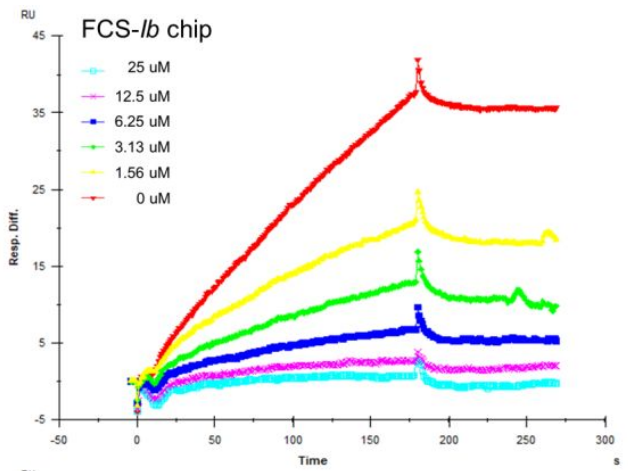

(b)

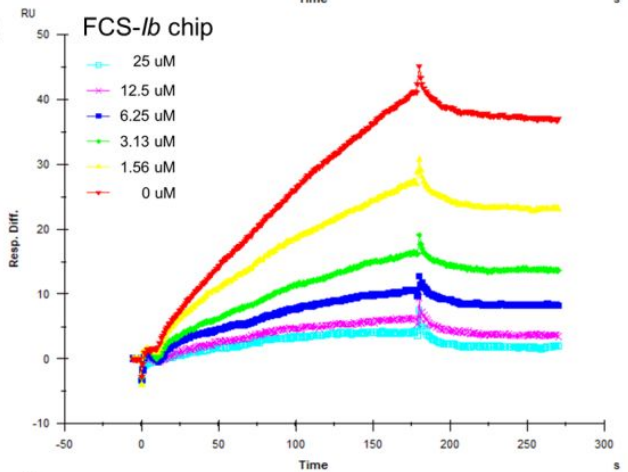

(c)

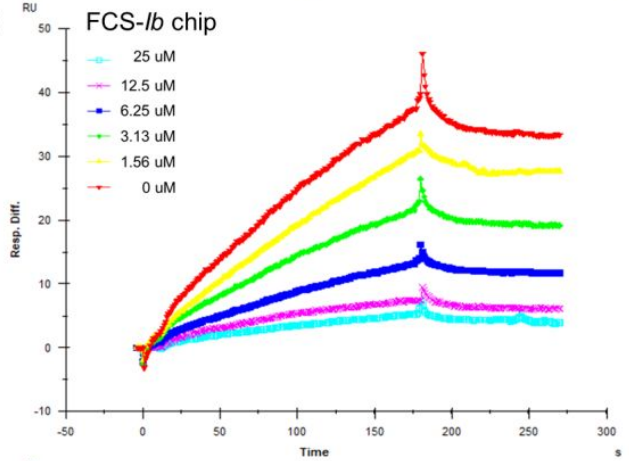

(d)

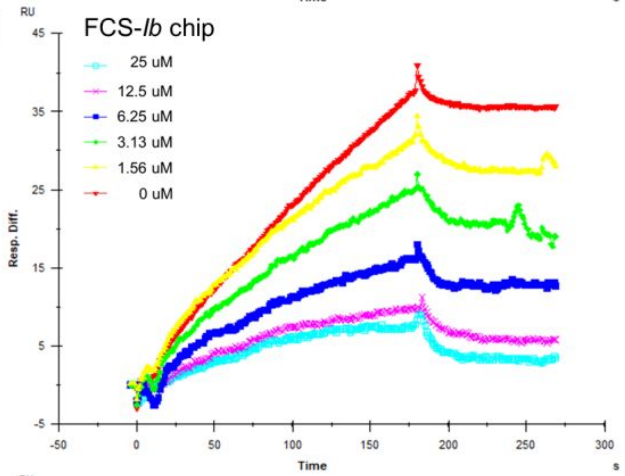

(e) ${ }^{R U}$, FCS-Ib chip

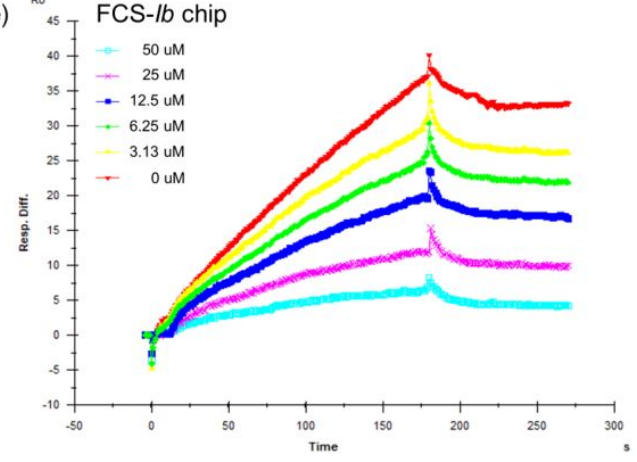

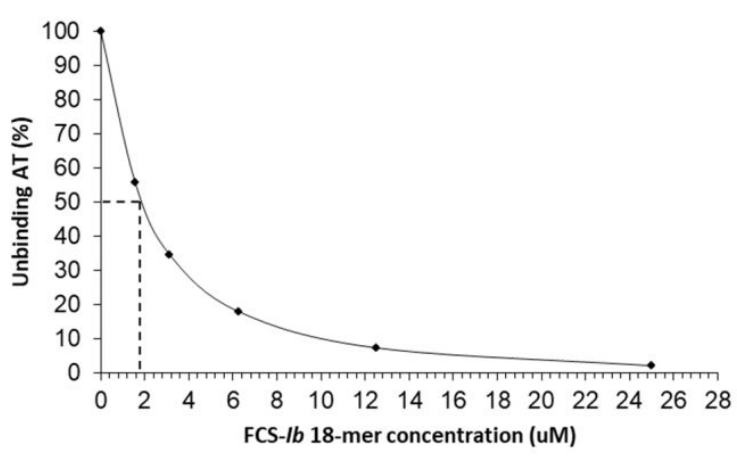
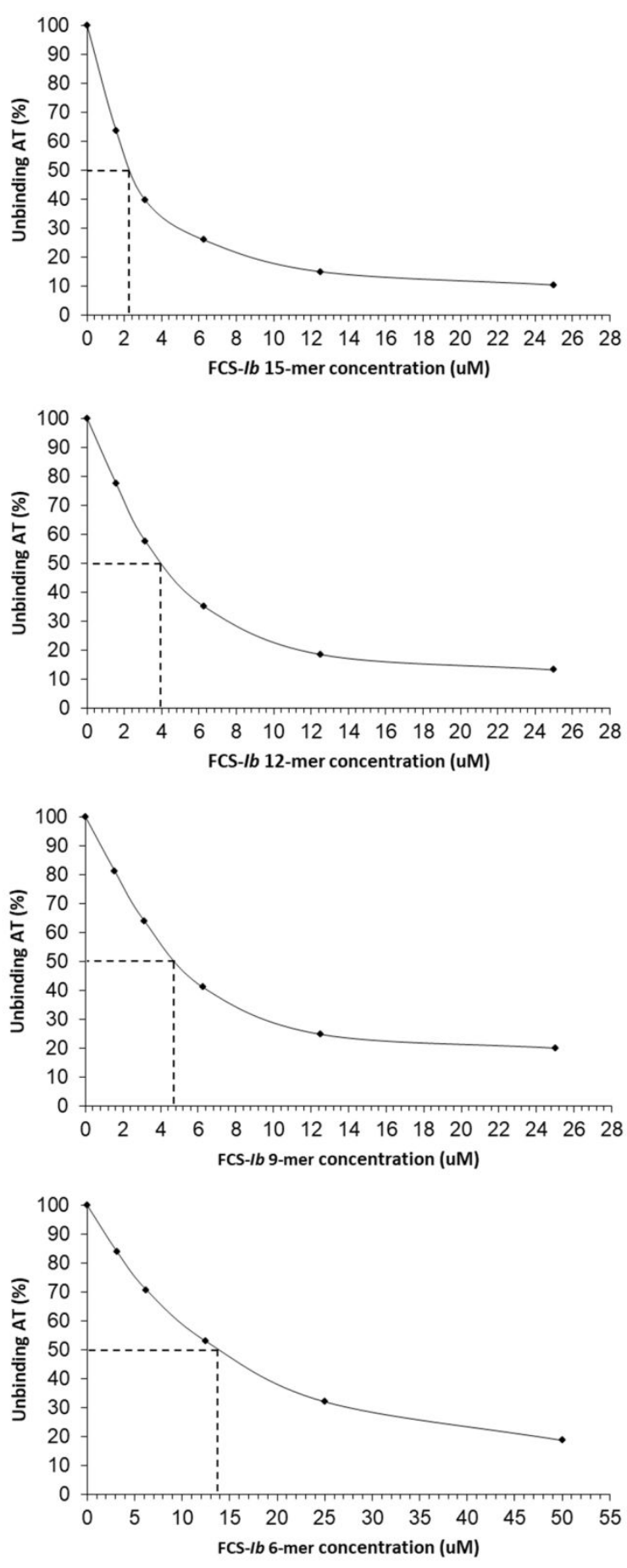
(f)

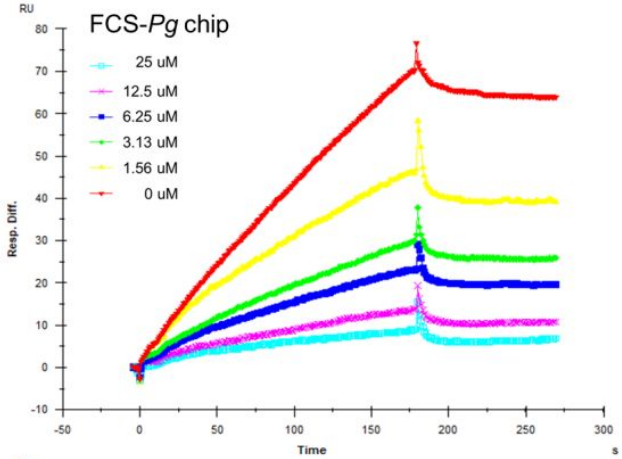

(g)

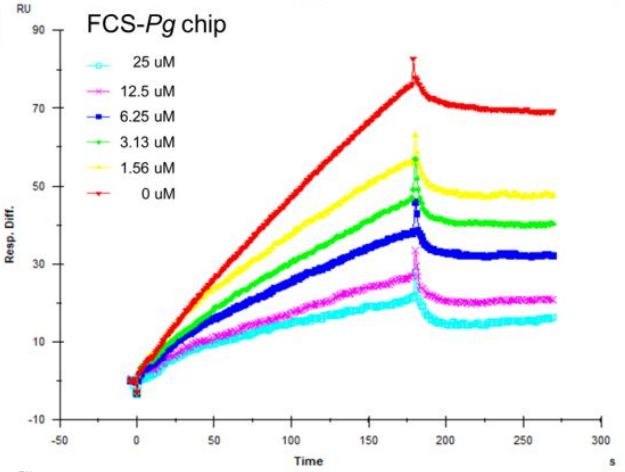

(h)

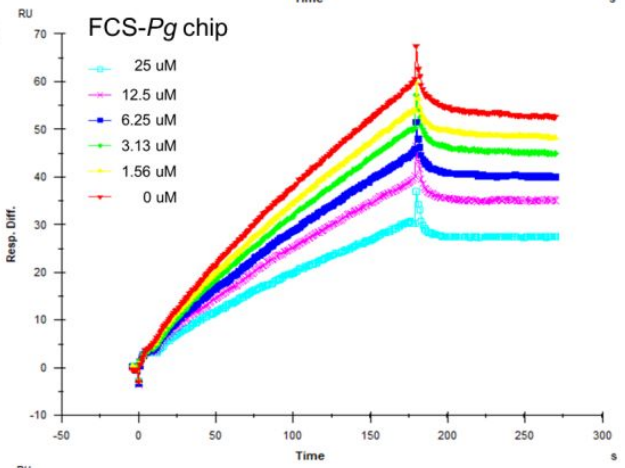

(i)

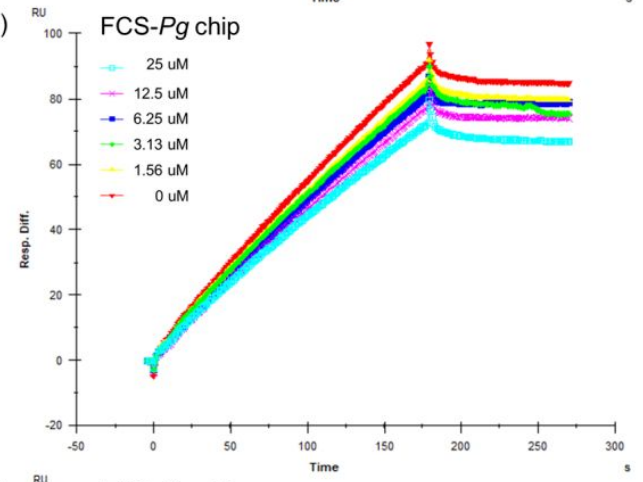

(j)

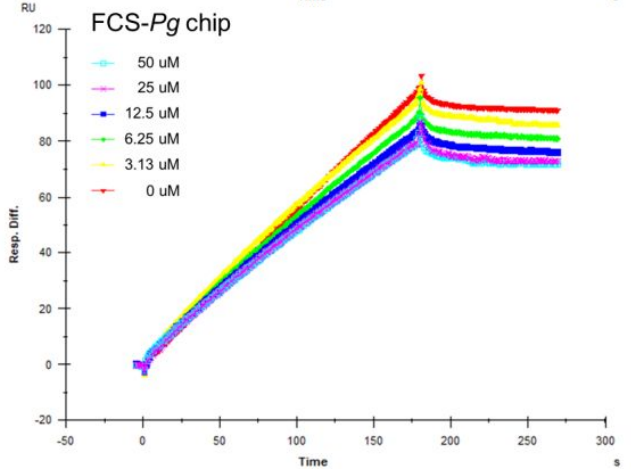

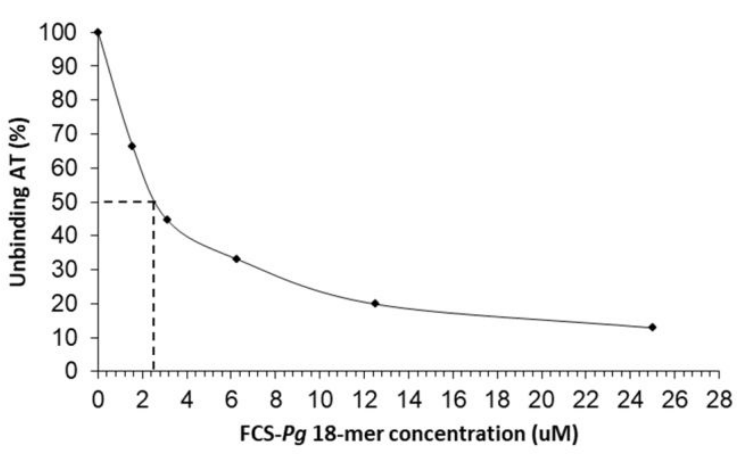
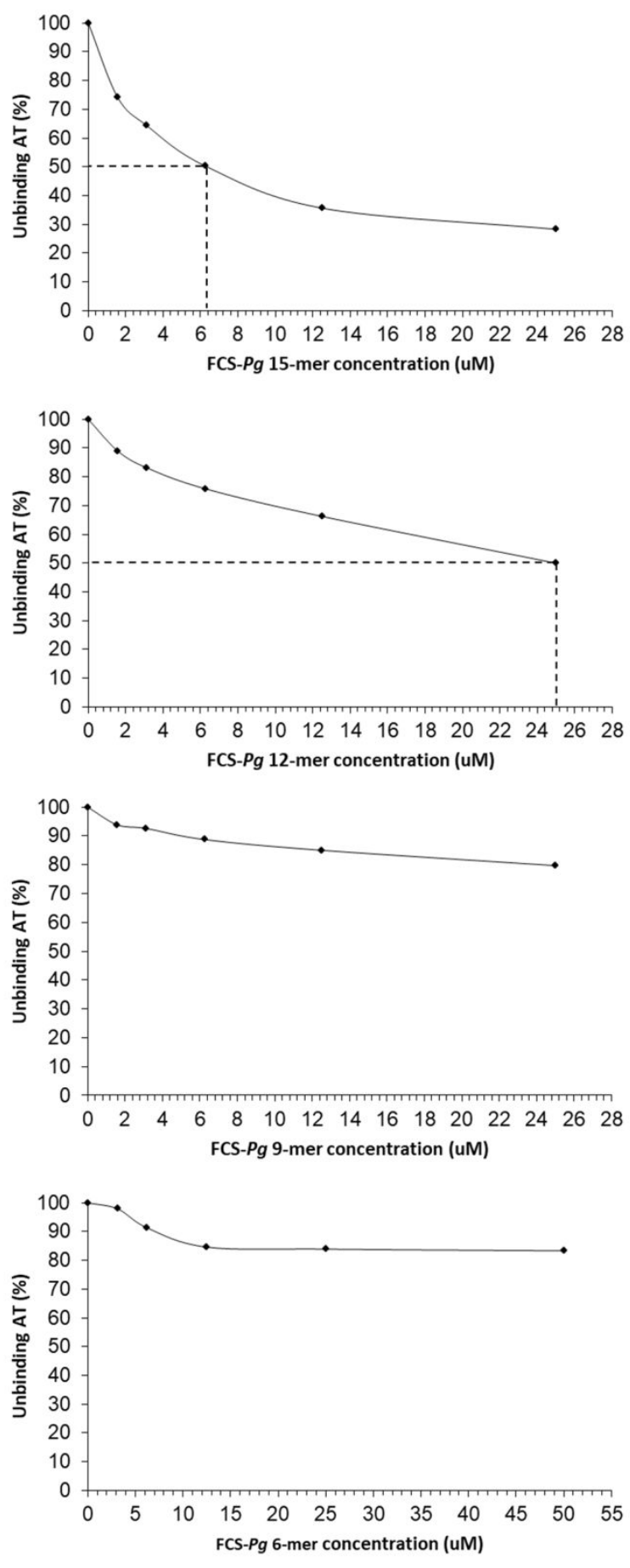

Figure S14. Competition of AT (500 nM) binding to immobilized FCS-Ib by gradient 
concentrations of (a) FCS-Ib 18-mer, (b) FCS-Ib 15-mer, (c) FCS-Ib 12-mer, (d) FCS-Ib 9-mer and (e) FCS-Ib 6-mer with corresponding $\mathrm{IC}_{50}$. Competition of AT (500 nM) binding to immobilized FCS- $P g$ by gradient concentrations of (f) FCS- $P g$ 18-mer, (g) FCS-Pg 15-mer, (h) FCS-Pg 12-mer, (i) FCS-Pg 9-mer and (j) FCS-Pg 6-mer with corresponding $\mathrm{IC}_{50}$. AT was preincubated with gradient concentrations of FCS oligomer prior to interact with immobilized FCS. The unbinding AT was the one not inhibited by FCS oligomer during preincubation. Unbinding AT (\%) was calculated by the highest response unit (RU) ratio of with and without preincubated FCS oligomer. 
(a)

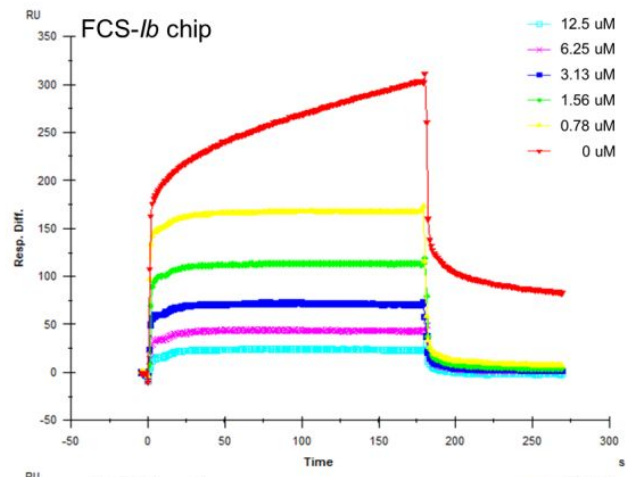

(b)

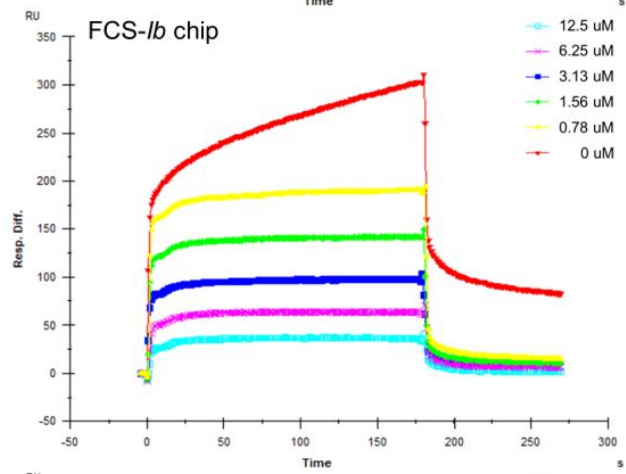

(c)

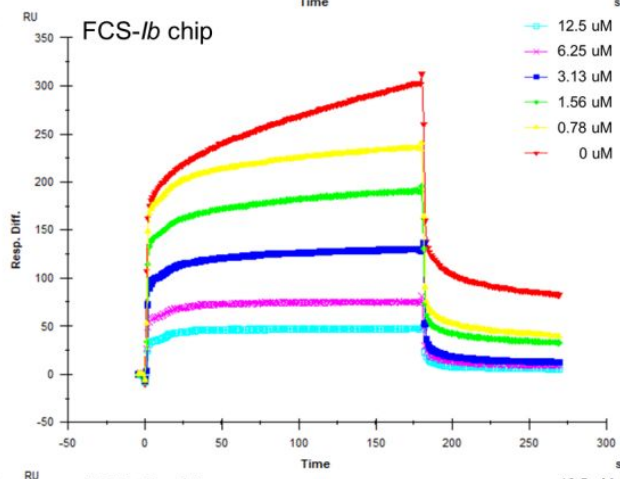

(d)

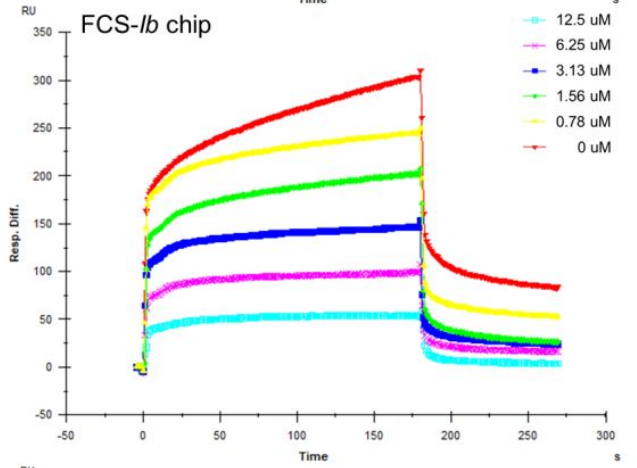

(e) ${ }_{350}^{\mathrm{RU}}$ FCS-lb chip $25 \mathrm{um}$

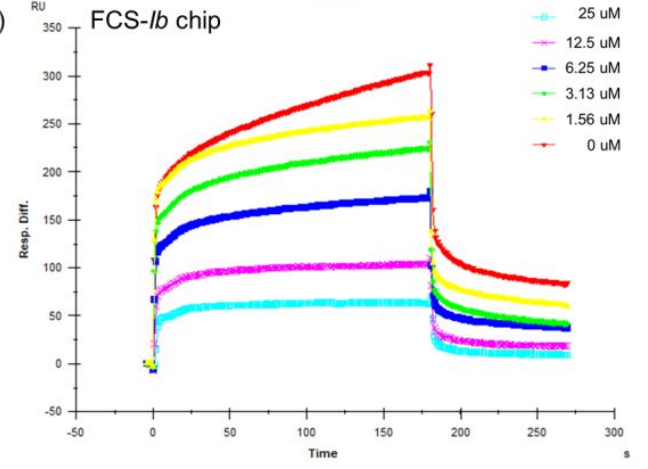

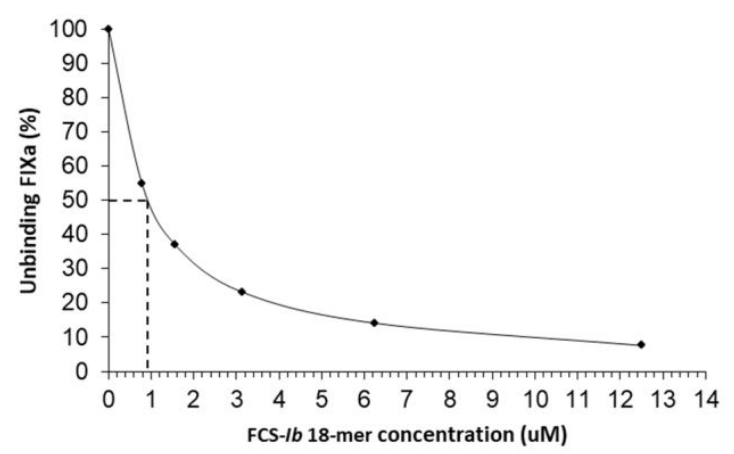
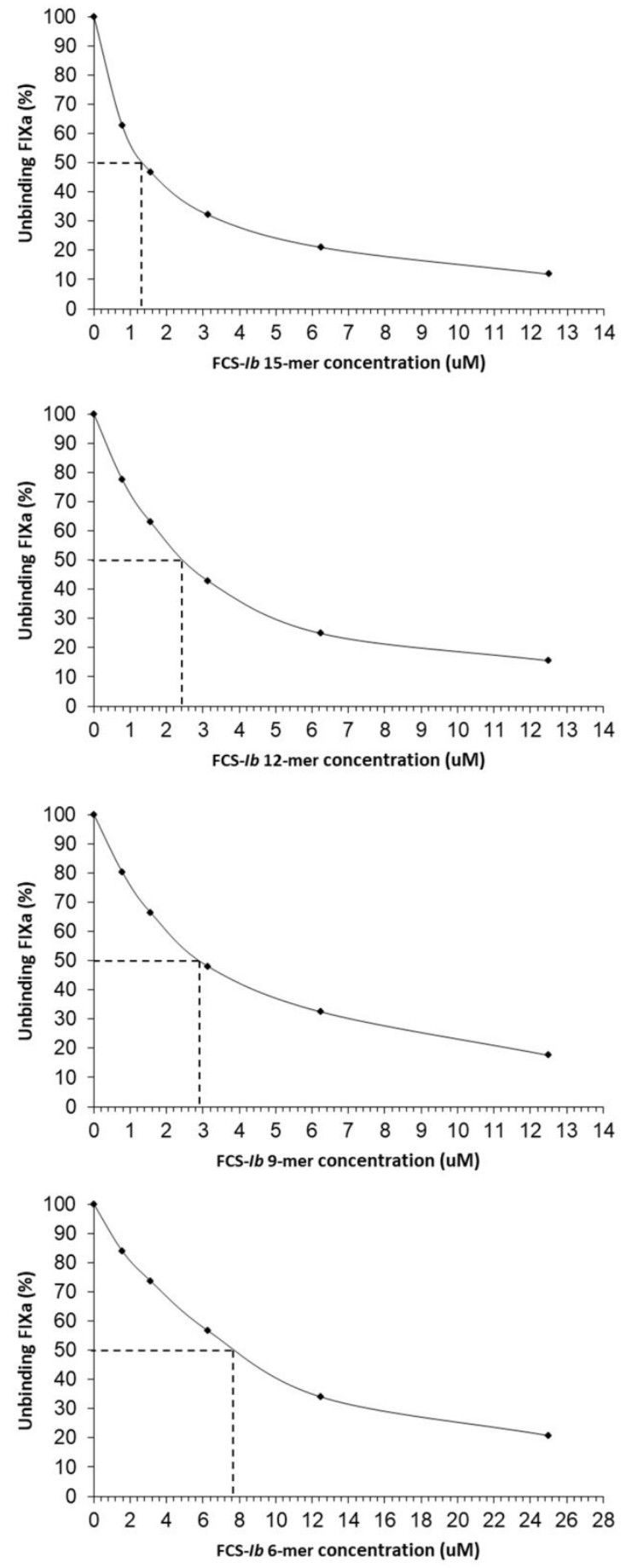
(f)

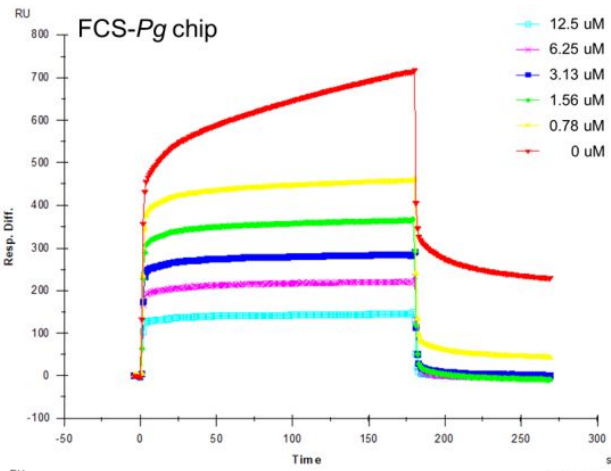

(g)

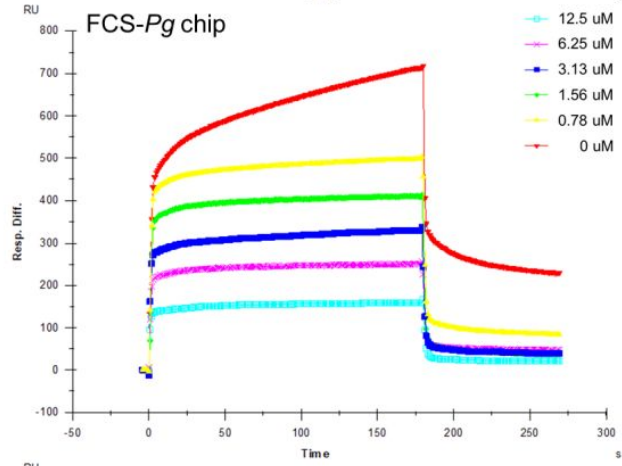

(h)

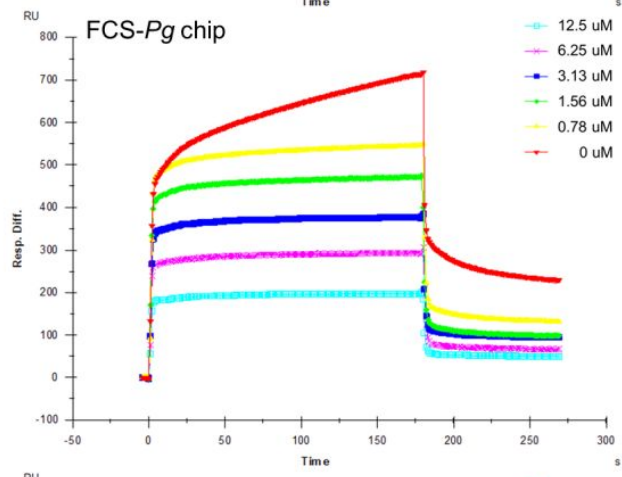

(i)

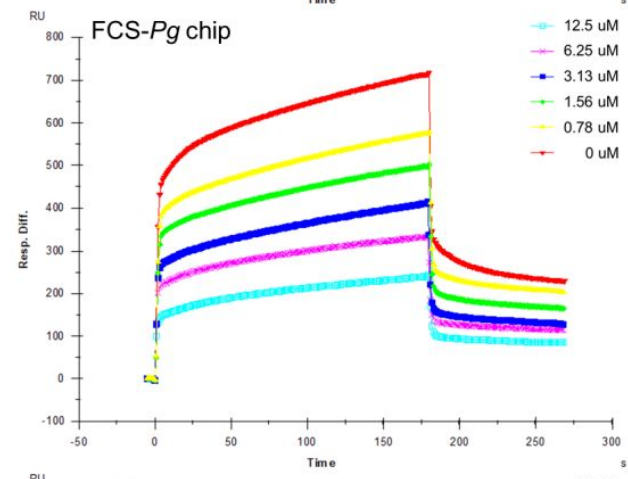

(j)

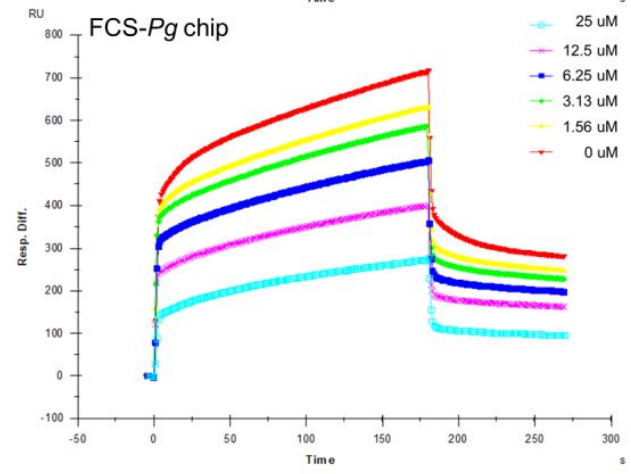

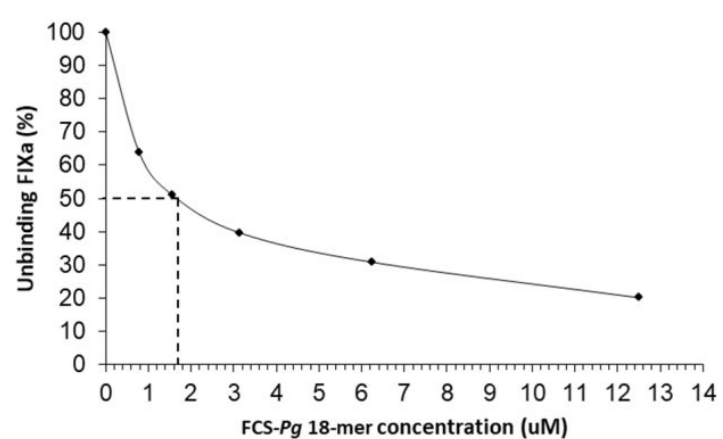
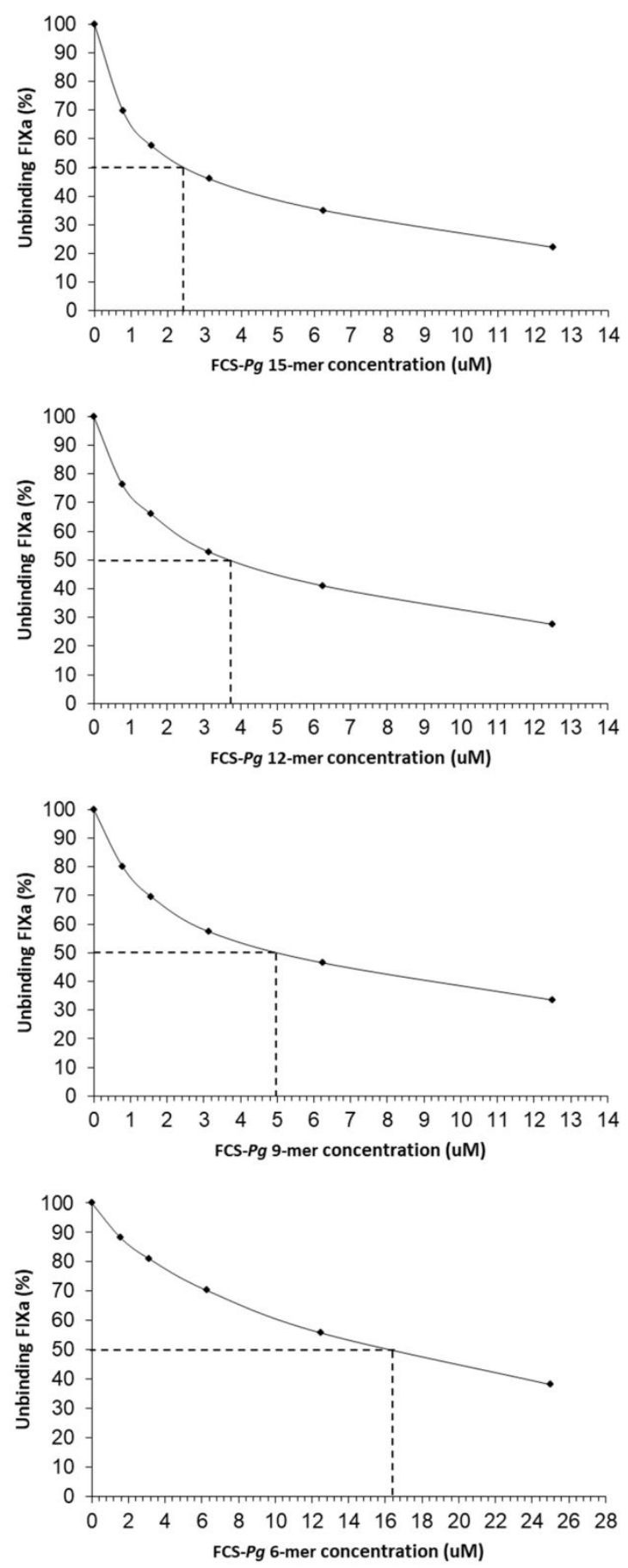

Figure S15. Competition of FIXa $(500 \mathrm{nM})$ binding to immobilized FCS-Ib by 
gradient concentrations of (a) FCS-Ib 18-mer, (b) FCS-Ib 15-mer, (c) FCS-Ib 12-mer, (d) FCS-Ib 9-mer and (e) FCS-Ib 6-mer with corresponding IC $_{50}$. Competition of FIXa (500 nM) binding to immobilized FCS-Pg by gradient concentrations of (f) FCS-Pg 18-mer, (g) FCS-Pg 15-mer, (h) FCS-Pg 12-mer, (i) FCS-Pg 9-mer and (j) FCS-Pg 6-mer with corresponding $\mathrm{IC}_{50}$. FIXa was preincubated with gradient concentrations of FCS oligomer prior to interact with immobilized FCS. The unbinding FIXa was the one not inhibited by FCS oligomer during preincubation. Unbinding FIXa (\%) was calculated by the highest response unit (RU) ratio of with and without preincubated FCS oligomer. 


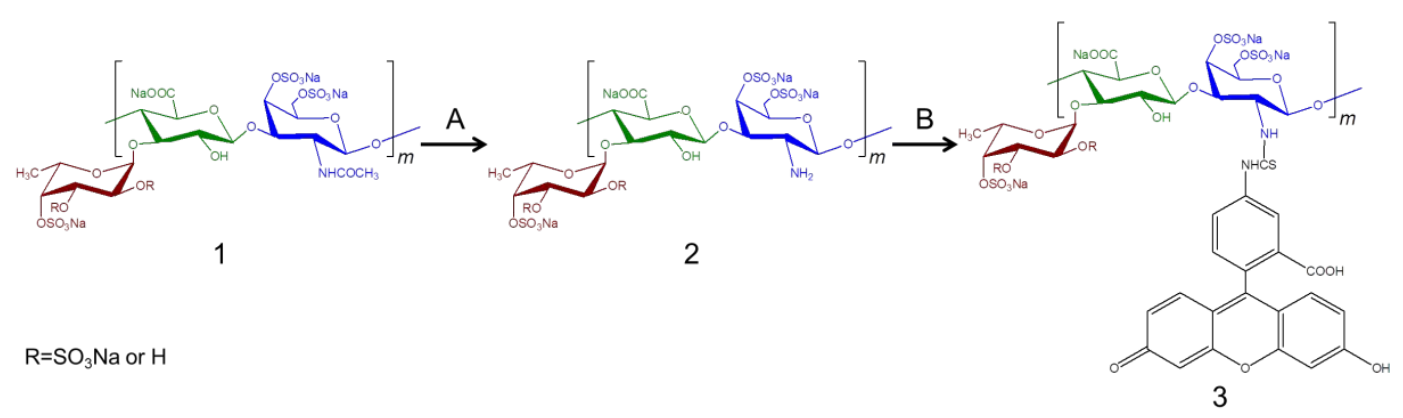

Figure S16. Preparation of FCS oligomers-FITC. (A) Two types of FCS 6-18 oligomers (compound 1) were mildly partial $N$-deacetylated with hydrazine hydrate. (B) The partial $\mathrm{N}$-deacetylated FCS oligomers (compound 2) were then labeled with FITC to get FCS oligomers-FITC (compound 3). Briefly, $100 \mathrm{mg}$ compound 2 and 20 mg FITC were dissolved in $5 \mathrm{~mL} 0.5 \mathrm{M} \mathrm{Na}_{2} \mathrm{CO}_{3}(\mathrm{pH} \mathrm{8.3).} \mathrm{Then,} \mathrm{the} \mathrm{mixture} \mathrm{was}$ removed from light and stirred at $25{ }^{\circ} \mathrm{C}$ for $24 \mathrm{~h}$. Finally, the product was dialyzed and lyophilized to remove the salt and get FITC labeled FCS oligomers. 


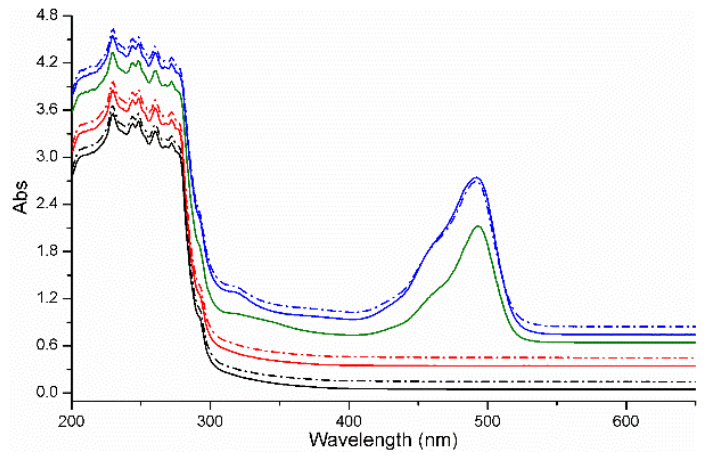

Figure S17. UV scanning spectra of the original FCS oligomers (black lines), partial $N$-deacetylated FCS oligomers (red lines), FITC labeled FCS oligomers (blue lines) and FITC (green line). The solid lines and dash dot lines indicate FCS-Ib oligomers and FCS-Pg oligomers, respectively. 

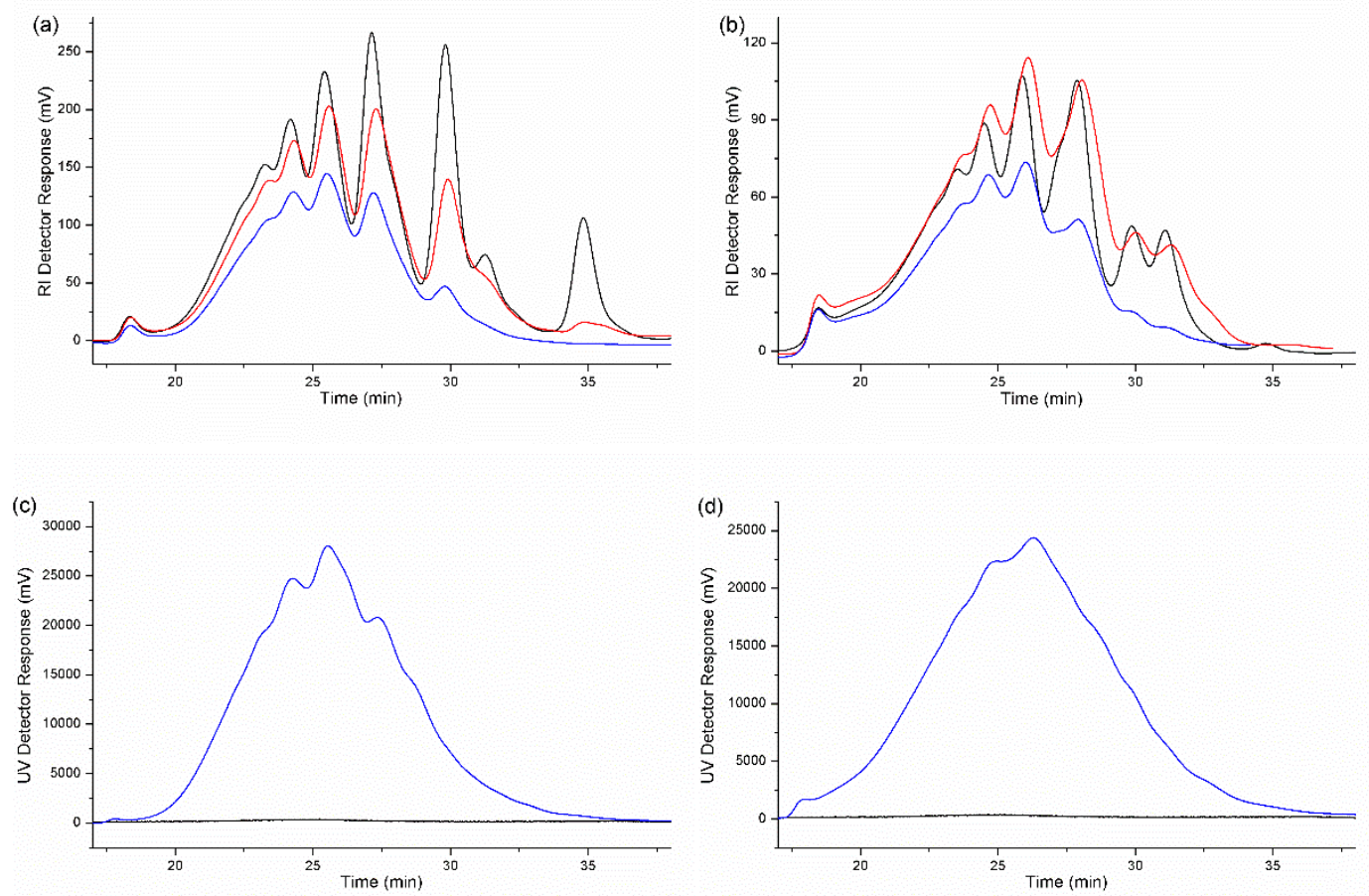

Figure S18. HPGPC profiles of (a) FCS-Ib oligomers and (b) FCS-Pg oligomers by refractive index detector. HPGPC profiles of (c) FCS-Ib oligomers and (d) FCS- $P g$ oligomers by UV detector at $490 \mathrm{~nm}$. The black lines, red lines and blue lines indicate the original samples, partial $N$-deacetylated samples and FITC labeled samples, respectively. 

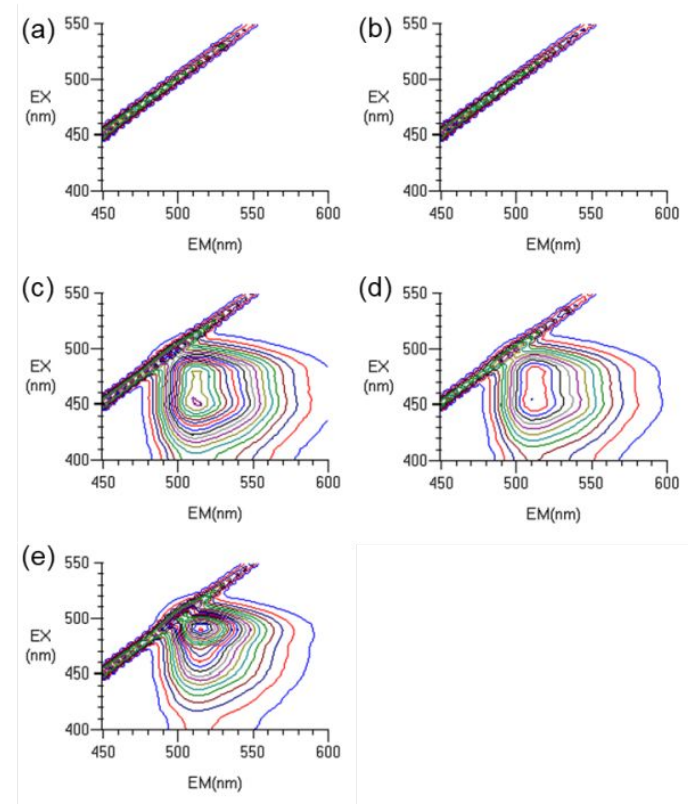

Figure S19. Fluorescence scanning spectra of (a) partial $N$-deacetylated FCS- $I b$ oligomers, (b) partial $N$-deacetylated FCS- $P g$ oligomers, (c) FCS-Ib oligomers-FITC, (d) FCS-Pg oligomers-FITC and (e) FITC. 